\title{
On the Boundedness of Biparameter Littlewood-Paley $g_{\lambda}^{*}$-Function
}

\begin{abstract}
Mingming Cao and Qingying Xue
School of Mathematical Sciences, Beijing Normal University, Laboratory of Mathematics and Complex Systems, Ministry of Education, Beijing 100875, China
\end{abstract}

Correspondence should be addressed to Qingying Xue; qyxue@bnu.edu.cn

Received 5 September 2016; Accepted 19 October 2016

Academic Editor: Dashan Fan

Copyright (C) 2016 M. Cao and Q. Xue. This is an open access article distributed under the Creative Commons Attribution License, which permits unrestricted use, distribution, and reproduction in any medium, provided the original work is properly cited.

Let $m, n \geq 1$ and let $g_{\lambda_{1}, \lambda_{2}}^{*}$ be the biparameter Littlewood-Paley $g_{\lambda}^{*}$-function defined by $g_{\lambda_{1}, \lambda_{2}}^{*}(f)(x)=\left(\iint_{\mathbb{R}_{+}^{m+1}}\left(t_{2} /\left(t_{2}+\right.\right.\right.$ $\left.\left.\left.\left|x_{2}-y_{2}\right|\right)\right)^{m \lambda_{2}} \iint_{\mathbb{R}_{+}^{n+1}}\left(t_{1} /\left(t_{1}+\left|x_{1}-y_{1}\right|\right)\right)^{n \lambda_{1}} \times\left|\theta_{t_{1}, t_{2}} f\left(y_{1}, y_{2}\right)\right|^{2}\left(d y_{1} d t_{1} / t_{1}^{n+1}\right)\left(d y_{2} d t_{2} / t_{2}^{m+1}\right)\right)^{1 / 2}, \lambda_{1}>1, \lambda_{2}>1$, where $\theta_{t_{1}, t_{2}} f$ is a nonconvolution kernel defined on $\mathbb{R}^{m+n}$. In this paper we show that the biparameter Littlewood-Paley function $g_{\lambda_{1}, \lambda_{2}}^{*}$ is bounded from $L^{2}\left(\mathbb{R}^{n+m}\right)$ to $L^{2}\left(\mathbb{R}^{n+m}\right)$. This is done by means of probabilistic methods and by using a new averaging identity over good double Whitney regions.

\section{Introduction}

1.1. Background and Motivation. It is well known that $g_{\lambda}^{*}-$ function originated in the work of Littlewood and Paley [1] in the 1930s. In 1961, Stein [2] introduced and studied the following higher dimensional $(n \geq 2)$ Littlewood-Paley $g_{\lambda}^{*}$ function:

$$
\begin{aligned}
& g_{\lambda}^{*}(f)(x) \\
& \quad=\left(\iint_{\mathbb{R}_{+}^{n+1}}\left(\frac{t}{t+|x-y|}\right)^{n \lambda}\left|\nabla P_{t} f(y, t)\right|^{2} \frac{d y d t}{t^{n-1}}\right)^{1 / 2},
\end{aligned}
$$

$$
\lambda>1
$$

where $P_{t} f(y, t)=p_{t} * f(y), p_{t}(y)=t^{-n} p(y / t)$ denotes the Poisson kernel, and $\nabla=\left(\partial / \partial y_{1}, \ldots, \partial / \partial y_{n}, \partial / \partial t\right)$. It plays important roles in harmonic analysis and other fields. It is easy to show that $g_{\lambda}^{*}$ is an isometry on $L^{2}\left(\mathbb{R}^{n}\right)$. With much greater difficulty, it can be proved that, for any $1<p<$ $\infty,\left\|g_{\lambda}^{*}(f)\right\|_{L^{p}\left(\mathbb{R}^{n}\right)}$ and $\|f\|_{L^{p}\left(\mathbb{R}^{n}\right)}$ are equivalent norms [3]. Moreover, in [3], Stein also proved that if $\lambda>2$ then $g_{\lambda}^{*}$ is of weak type $(1,1)$ and is of strong type $(p, p)$ for $1<p<\infty$. In 1970, as a replacement of weak $(1,1)$ bounds for $1<\lambda<2$, Fefferman [4] considered the endpoint weak $(p, p)$ estimates of $g_{\lambda}^{*}$-function when $p>1$ and $\lambda=2 / p$.
Recently, Cao et al. [5] gave a characterization of twoweight norm inequalities for the classical $g_{\lambda}^{*}$-function. The first step of the proof is to reduce the case to good Whitney regions. In addition, the random dyadic grids and martingale differences decomposition are used. The core of the proof is the construction of stopping cubes, which is a modern and effective technique to deal with two-weight problems. The stopping cubes were first introduced to handle two-weight boundedness for Hilbert transform $[6,7]$. Then the related consequences and applications were given, as demonstrated in $[5,8,9]$. Still, more recently, Cao and Xue [10] established a local $\mathrm{Tb}$ theorem for the nonhomogeneous LittlewoodPaley $g_{\lambda}^{*}$-function with nonconvolution type kernels and upper power bound measure $\mu$. It was the first time to investigate $g_{\lambda}^{*}$-function in the simultaneous presence of three attributes: local, nonhomogeneous, and $L^{p}$-testing condition. It is important to note that the testing condition here is $L^{p}$ type with $p \in(1,2]$, which means that the averaging identity over good Whitney regions used in [5] is not suitable for the new setting $p \in(1,2)$. Thus, some new methods and more complicated techniques are needed.

When it comes to the multiparameter harmonic analysis, there is a very large existing theory. In terms of singular integrals, it was initiated in the work of Fefferman and Stein [11] on biparameter singular integral operators and then 
continued by many authors. In 2012, a dyadic representation theorem for biparameter singular integrals was presented by Martikainen [12]. As a consequence, a new version of the product space $T 1$ theorem was established. In 2014, Hytönen and Martikainen [13] proved a nonhomogeneous version of $T 1$ theorem for certain biparameter singular integral operators. Moreover, they discussed the related nonhomogeneous Journés lemma and product BMO theory with more general type of measures. Still, in 2014, a class of biparameter kernels and related vertical square functions in the upper half-space were first introduced by Martikainen [14]. Using modern dyadic probabilistic techniques adapted to the biparameter situation, the author gave a criterion for the $L^{2}\left(\mathbb{R}^{n+m}\right)$ boundedness of these square functions. It is worth pointing out that the kernels are assumed to satisfy some estimates, including a natural size condition, a Hölder estimate and two symmetric mixed Hölder and size estimates, the mixed Carleson and size conditions, the mixed Carleson and Hölder estimates, and a biparameter Carleson condition. Moreover, it should be noted that the biparameter Carleson condition is necessary for the square function to be bounded in $L^{2}\left(\mathbb{R}^{n+m}\right)$.

Motivated by the above works, in this paper, we keep on studying the Littlewood-Paley $g_{\lambda}^{*}$-function but in biparameter setting. To state more clearly, we first introduce the definition of the biparameter Littlewood-Paley $g_{\lambda}^{*}$-function.

Definition 1. Let $\lambda_{1}, \lambda_{2}>1$, for any $x=\left(x_{1}, x_{2}\right) \in \mathbb{R}^{n+m}$, and the biparameter Littlewood-Paley $g_{\lambda}^{*}$-function is defined by

$$
\begin{aligned}
& g_{\lambda_{1}, \lambda_{2}}^{*}(f)(x):=\left(\iint_{\mathbb{R}_{+}^{m+1}}\left(\frac{t_{2}}{t_{2}+\left|x_{2}-y_{2}\right|}\right)^{m \lambda_{2}}\right. \\
& \cdot \iint_{\mathbb{R}_{+}^{n+1}}\left(\frac{t_{1}}{t_{1}+\left|x_{1}-y_{1}\right|}\right)^{n \lambda_{1}} \\
& \left.\quad \times\left|\theta_{t_{1}, t_{2}} f\left(y_{1}, y_{2}\right)\right|^{2} \frac{d y_{1} d t_{1}}{t_{1}^{n+1}} \frac{d y_{2} d t_{2}}{t_{2}^{m+1}}\right)^{1 / 2},
\end{aligned}
$$

where $\theta_{t_{1}, t_{2}} f\left(y_{1}, y_{2}\right)=\iint_{\mathbb{R}^{n+m}} K_{t_{1}, t_{2}}\left(y_{1}, y_{2}, z_{1}, z_{2}\right) f\left(z_{1}\right.$, $\left.z_{2}\right) d z_{1} d z_{2}$.

Under certain structural assumptions, we will prove the following $L^{2}\left(\mathbb{R}^{n+m}\right)$ boundedness of $g_{\lambda_{1}, \lambda_{2}}^{*}$, in other words, the following inequality:

$$
\left\|g_{\lambda_{1}, \lambda_{2}}^{*}(f)\right\|_{L^{2}\left(\mathbb{R}^{n+m}\right)} \lesssim\|f\|_{L^{2}\left(\mathbb{R}^{n+m}\right)} .
$$

Compared to the biparameter vertical square function, the biparameter Littlewood-Paley $g_{\lambda}^{*}$-function is significantly much more difficult to be dealt with. Actually, in biparameter case, additional integrals make most of the corresponding estimates more complicated. We could not use the assumptions in [14] directly, since addition terms appear in Definition 1 . In fact, we will use much more weaker conditions than the conditions used in [14] (see assumptions in the following subsection). Unlike the one-parameter case and twoweight case [5], the proof of biparameter $g_{\lambda}^{*}$-function does not involve the stopping cubes and martingale differences decomposition. In fact, the decomposition associated with Haar function in $\mathbb{R}^{n}$ provides a foundation for our analysis. And modern techniques, including probabilistic methods and dyadic analysis, will be used efficiently again. They were first used by Martikainen [12] in the study of the biparameter Calderón-Zygmund integrals and later appeared in [14]. For more applications, one can refer to $[13,15]$.

1.2. Assumptions and Main Result. To state our main results, the natural framework is to give some appropriate assumptions. From now on, we always assume that $\alpha, \beta>0$. We use, for minor convenience, $\ell^{\infty}$ metrics on $\mathbb{R}^{n}$ and $\mathbb{R}^{m}$.

Assumption 1 (standard estimates). The kernel $K_{t_{1}, t_{2}}: \mathbb{R}^{n+m} \times$ $\mathbb{R}^{n+m} \rightarrow \mathbb{C}$ is assumed to satisfy the following estimates:

(1) Size condition:

$$
\begin{aligned}
& \left|K_{t_{1}, t_{2}}(x, y)\right| \\
& \quad \leq \frac{t_{1}^{\alpha}}{\left(t_{1}+\left|x_{1}-y_{1}\right|\right)^{n+\alpha}} \frac{t_{2}^{\beta}}{\left(t_{2}+\left|x_{2}-y_{2}\right|\right)^{m+\beta}} .
\end{aligned}
$$

(2) Hölder condition:

$$
\begin{aligned}
& \mid K_{t_{1}, t_{2}}(x, y)-K_{t_{1}, t_{2}}\left(x,\left(y_{1}, y_{2}^{\prime}\right)\right)-K_{t_{1}, t_{2}}\left(x,\left(y_{1}^{\prime}, y_{2}\right)\right) \\
& +K_{t_{1}, t_{2}}\left(x, y^{\prime}\right) \mid \lesssim \frac{\left|y_{1}-y_{1}^{\prime}\right|^{\alpha}}{\left(t_{1}+\left|x_{1}-y_{1}\right|\right)^{n+\alpha}} \\
& \quad \cdot \frac{\left|y_{2}-y_{2}^{\prime}\right|^{\beta}}{\left(t_{2}+\left|x_{2}-y_{2}\right|\right)^{m+\beta}},
\end{aligned}
$$$$
\text { whenever }\left|y_{1}-y_{1}^{\prime}\right|<t_{1} / 2 \text { and }\left|y_{2}-y_{2}^{\prime}\right|<t_{2} / 2 \text {. }
$$

(3) Mixed Hölder and size conditions:

$$
\begin{aligned}
& \left|K_{t_{1}, t_{2}}(x, y)-K_{t_{1}, t_{2}}\left(x,\left(y_{1}, y_{2}^{\prime}\right)\right)\right| \\
& \quad \lesssim \frac{t_{1}^{\alpha}}{\left(t_{1}+\left|x_{1}-y_{1}\right|\right)^{n+\alpha}} \frac{\left|y_{2}-y_{2}^{\prime}\right|^{\beta}}{\left(t_{2}+\left|x_{2}-y_{2}\right|\right)^{m+\beta}},
\end{aligned}
$$

whenever $\left|y_{2}-y_{2}^{\prime}\right|<t_{2} / 2$ and

$$
\begin{aligned}
& \left|K_{t_{1}, t_{2}}(x, y)-K_{t_{1}, t_{2}}\left(x,\left(y_{1}^{\prime}, y_{2}\right)\right)\right| \\
& \quad \lesssim \frac{\left|y_{1}-y_{1}^{\prime}\right|^{\alpha}}{\left(t_{1}+\left|x_{1}-y_{1}\right|\right)^{n+\alpha}} \frac{t_{2}^{\beta}}{\left(t_{2}+\left|x_{2}-y_{2}\right|\right)^{m+\beta}},
\end{aligned}
$$

whenever $\left|y_{1}-y_{1}^{\prime}\right|<t_{1} / 2$.

Assumption 2 (Carleson condition $\times$ standard estimates). If $I \subset \mathbb{R}^{n}$ is a cube with side length $\ell(I)$, we define the associated Carleson box by $\widehat{I}=I \times(0, \ell(I))$. We assume the following conditions: for every cube $I \subset \mathbb{R}^{n}$ and $J \subset \mathbb{R}^{m}$, it holds the following: 
(1) Combinations of Carleson and size conditions:

$$
\begin{gathered}
\left(\iint_{\widehat{J}} \int_{\mathbb{R}^{m}}\left|\int_{J} K_{t_{1}, t_{2}}\left(x-y, z_{1}, z_{2}\right) d z_{2}\right|^{2}\right. \\
\left.\cdot\left(\frac{t_{2}}{t_{2}+\left|y_{2}\right|}\right)^{n \lambda_{1}} \frac{d y_{2}}{t_{2}^{n}} \frac{d x_{2} d t_{2}}{t_{2}}\right)^{1 / 2} \\
\quad \leq|J|^{1 / 2} \frac{t_{1}^{\alpha}}{\left(t_{1}+\left|x_{1}-y_{1}-z_{1}\right|\right)^{n+\alpha}} .
\end{gathered}
$$$$
\left(\iint_{\widehat{I}} \int_{\mathbb{R}^{n}}\left|\int_{I} K_{t_{1}, t_{2}}\left(x-y, z_{1}, z_{2}\right) d z_{1}\right|^{2}\right.
$$$$
\left.\cdot\left(\frac{t_{1}}{t_{1}+\left|y_{1}\right|}\right)^{n \lambda_{1}} \frac{d y_{1}}{t_{1}^{n}} \frac{d x_{1} d t_{1}}{t_{1}}\right)^{1 / 2}
$$$$
\lesssim|I|^{1 / 2} \frac{t_{2}^{\beta}}{\left(t_{2}+\left|x_{2}-y_{2}-z_{2}\right|\right)^{m+\beta}},
$$

(2) Combinations of Carleson and Hölder conditions:

$$
\begin{aligned}
& \left(\iint_{\widehat{I}} \int_{\mathbb{R}^{n}}\left|\int_{I}\left[K_{t_{1}, t_{2}}\left(x-y, z_{1}, z_{2}\right)-K_{t_{1}, t_{2}}\left(x-y, z_{1}, z_{2}^{\prime}\right)\right] d z_{1}\right|^{2}\left(\frac{t_{1}}{t_{1}+\left|y_{1}\right|}\right)^{n \lambda_{1}} \frac{d y_{1}}{t_{1}^{n}} \frac{d x_{1} d t_{1}}{t_{1}}\right)^{1 / 2} \lesssim|I|^{1 / 2} \\
& \quad \cdot \frac{\left|z_{2}-z_{2}^{\prime}\right|^{\beta}}{\left(t_{2}+\left|x_{2}-y_{2}-z_{2}\right|\right)^{m+\beta}},
\end{aligned}
$$

whenever $\left|z_{2}-z_{2}^{\prime}\right|<t_{2} / 2$. And

$$
\begin{aligned}
& \left(\iint_{\widehat{J}} \int_{\mathbb{R}^{m}}\left|\int_{J}\left[K_{t_{1}, t_{2}}\left(x-y, z_{1}, z_{2}\right)-K_{t_{1}, t_{2}}\left(x-y, z_{1}^{\prime}, z_{2}\right)\right] d z_{2}\right|^{2}\left(\frac{t_{2}}{t_{2}+\left|y_{2}\right|}\right)^{m \lambda_{2}} \frac{d y_{2}}{t_{2}^{n}} \frac{d x_{2} d t_{2}}{t_{2}}\right)^{1 / 2} \leqslant|J|^{1 / 2} \\
& \quad \cdot \frac{\left|z_{1}-z_{1}^{\prime}\right|^{\alpha}}{\left(t_{1}+\left|x_{1}-y_{1}-z_{1}\right|\right)^{n+\alpha}}
\end{aligned}
$$

whenever $\left|z_{1}-z_{1}^{\prime}\right|<t_{1} / 2$.

Assumption 3 (biparameter Carleson condition). Let $\mathscr{D}=$ $\mathscr{D}_{n} \times \mathscr{D}_{m}$, where $\mathscr{D}_{n}$ is a dyadic grid in $\mathbb{R}^{n}$ and $\mathscr{D}_{m}$ is a dyadic grid in $\mathbb{R}^{m}$. For $I \in \mathscr{D}_{n}$, let $W_{I}=I \times(\ell(I) / 2, \ell(I))$ be the associated Whitney region. Denote $n_{1}=n, n_{2}=m$, and

$$
\begin{aligned}
C_{I J}^{D} & =\iint_{W_{J}} \iint_{W_{I}} \iint_{\mathbb{R}^{n+m}}\left|\theta_{t_{1}, t_{2}} \mathbf{1}\left(y_{1}, y_{2}\right)\right|^{2} \\
. & {\left[\prod_{i=1}^{2}\left(\frac{t_{i}}{t_{i}+\left|x_{i}-y_{i}\right|}\right)^{n_{i} \lambda_{i}}\right] \frac{d y_{1} d y_{2}}{t_{1}^{n} t_{2}^{m}} \frac{d x_{1} d t_{1}}{t_{1}} \frac{d x_{2} d t_{2}}{t_{2}} . }
\end{aligned}
$$

We assume the following biparameter Carleson condition: for every $\mathscr{D}=\mathscr{D}_{n} \times \mathscr{D}_{m}$ it holds that

$$
\sum_{\substack{I \times J \in \mathscr{D} \\ I \times J \subset \Omega}} C_{I J}^{\mathscr{D}} \lesssim|\Omega|
$$

for all sets $\Omega \subset \mathbb{R}^{n+m}$ such that $|\Omega|<\infty$ and such that for every $x \in \Omega$ there exists $I \times J \in \mathscr{D}$ so that $x \in I \times J \subset \Omega$.

Now we state the main result of this paper.
Theorem 2. Let $\lambda_{1}, \lambda_{2}>2,0<\alpha \leq n\left(\lambda_{1}-2\right) / 2$, and $0<$ $\beta \leq m\left(\lambda_{2}-2\right) / 2$. Assume that the kernel $K_{t_{1}, t_{2}}$ satisfies the Assumptions 1-3. Then it holds that

$$
\left\|g_{\lambda_{1}, \lambda_{2}}^{*}(f)\right\|_{L^{2}\left(\mathbb{R}^{n+m}\right)} \leqslant\|f\|_{L^{2}\left(\mathbb{R}^{n+m}\right)},
$$

where the implied constant depends only on the assumptions.

Remark 3. In Section 6, we shall show that the biparameter Carleson condition is necessary for $g_{\lambda_{1}, \lambda_{2}}^{*}$-function bound on $L^{2}\left(\mathbb{R}^{n+m}\right)$. Moreover, Assumptions 2 and 3 are much weaker than the similar conditions used in [14], since here two terms (both less than one) were added and more integrals related to $y_{1}$ or $y_{2}$ were used in our assumptions.

\section{The Probabilistic Reduction}

In this section, our goal is to simplify the proof of the main result. First, we recall the definitions of random dyadic grids, good/bad cubes, Haar function on $\mathbb{R}^{n}$ which can be found in $[12,16,17]$. 
2.1. Random Dyadic Grids. Let $\beta_{n}=\left\{\beta_{n}^{j}\right\}_{j \in \mathbb{Z}}$, where $\beta_{n}^{j} \in$ $\{0,1\}^{n}$. Let $\mathscr{D}_{n}^{0}$ be the standard dyadic grids on $\mathbb{R}^{n}$. We define the new dyadic grid in $\mathbb{R}^{n}$ by

$$
\begin{aligned}
\mathscr{D}_{n} & =\left\{I+\beta_{n} ; I \in \mathscr{D}_{n}^{0}\right\} \\
& :=\left\{I+\sum_{j: 2^{-j}<\ell(I)} 2^{-j} \beta_{n}^{j} ; I \in \mathscr{D}_{n}^{0}\right\} .
\end{aligned}
$$

Similarly, we can define the dyadic grids $\mathscr{D}_{m}$ in $\mathbb{R}^{m}$. There is a natural product probability structure on $\left(\{0,1\}^{n}\right)^{\mathbb{Z}}$ and $\left(\{0,1\}^{m}\right)^{\mathbb{Z}}$. So we have independent random dyadic grids $\mathscr{D}_{n}$ and $\mathscr{D}_{m}$ in $\mathbb{R}^{n}$ and $\mathbb{R}^{m}$, respectively. Even if $n=m$ we need two independent grids.

2.2. Good and Bad Cubes. A cube $I \in \mathscr{D}_{n}$ is said to be bad if there exists a $J \in \mathscr{D}_{n}$ with $\ell(J) \geq 2^{r} \ell(I)$ such that $\operatorname{dist}(I, \partial J) \leq$ $\ell(I)^{\gamma_{n}} \ell(J)^{1-\gamma_{n}}$. Otherwise, $I$ is called good. Here $r \in \mathbb{Z}_{+}$and $\gamma_{n} \in(0,1 / 2)$ are given parameters. Denote $\pi_{\text {good }}^{n}=\mathbb{P}_{\beta_{n}}(I+$ $\beta_{n}$ is good $)=\mathbb{E}_{\beta_{n}}\left(\boldsymbol{1}_{\text {good }}\left(I+\beta_{n}\right)\right)$. Then $\pi_{\text {good }}^{n}$ is independent of $I \in \mathscr{D}_{n}^{0}$, and the parameter $r$ is a fixed constant so that $\pi_{\text {good }}^{n}, \pi_{\text {good }}^{m}>0$.

Throughout this article, we take $\gamma_{n}=\alpha / 2(n+\alpha)$, where $\alpha>0$ appears in the kernel estimates. Moreover, roughly speaking, a dyadic cube $I$ will be bad if it is relatively close to the boundary of a much bigger dyadic cube. It is important to observe that the position and goodness of a cube $I \in \mathscr{D}_{n}^{0}$ are independent. Indeed, according to the definition, the spatial position of

$$
I+\beta_{n}=I+\sum_{j: 2^{-j}<\ell(I)} 2^{-j} \beta_{n}^{j}
$$

depends only on $\beta_{n}^{j}$ for $2^{-j}<\ell(I)$. On the other hand, the relative position of $I+\beta_{n}$ with respect to a bigger cube

$$
J+\beta_{n}=I+\sum_{j: 2^{-j}<\ell(I)} 2^{-j} \beta_{n}^{j}+\sum_{j: \ell(I) \leq 2^{-j}<\ell(J)} 2^{-j} \beta_{n}^{j}
$$

depends only on $\beta_{n}^{j}$ for $\ell(I) \leq 2^{-j}<\ell(J)$. Thus, the position and goodness of $I+\beta_{n}$ are independent.

2.3. Haar Functions. In order to decompose a function $f \in$ $L^{2}$, we next recall the definition of the Haar function on $\mathbb{R}^{n}$. Let $h_{I}$ be an $L^{2}$ normalized Haar function related to $I \in \mathscr{D}_{n}$, where $\mathscr{D}_{n}$ is a dyadic grid on $\mathbb{R}^{n}$. With this we mean that $h_{I}, I=I_{1} \times \cdots \times I_{n}$, is one of the $2^{n}$ functions $h_{I}^{\eta}, \eta=$ $\eta_{1}, \ldots, \eta_{n} \in\{0,1\}^{n}$, defined by

$$
h_{I}^{\eta}=h_{I_{1}}^{\eta_{1}} \otimes \cdots \otimes h_{I_{n}}^{\eta_{n}},
$$

where $h_{I_{i}}^{0}=\left|I_{i}\right|^{-1 / 2} \mathbf{1}_{I_{i}}$ and $h_{I_{i}}^{1}=\left|I_{i}\right|^{-1 / 2}\left(\mathbf{1}_{I_{i, l}}-\mathbf{1}_{I_{i, r}}\right)$ for every $i=1, \ldots, n$. Here $I_{i, l}$ and $I_{i, r}$ are the left and right halves of the interval $I_{i}$, respectively. If $\eta \neq 0$, the Haar function is cancellative: $\int_{\mathbb{R}^{n}} h_{I}=0$. All the cancellative Haar functions form an orthonormal basis of $L^{2}\left(\mathbb{R}^{n}\right)$. If $a \in L^{2}\left(\mathbb{R}^{n}\right)$, we may thus write

$$
a=\sum_{I \in \mathscr{D}_{n}} \sum_{\eta \in\left\{0,1^{n} \backslash\{0\}\right.}\left\langle a, h_{I}^{\eta}\right\rangle h_{I}^{\eta}
$$

However, we suppress the finite $\eta$ summation and just write $a=\sum_{I}\left\langle a, h_{I}\right\rangle h_{I}$. We may expand a function $f$ defined in $\mathbb{R}^{n+m}$ using the corresponding product basis:

$$
f=\sum_{I, J} f_{I J} h_{I \times J}:=\sum_{I, J}\left\langle f, h_{I} \otimes h_{J}\right\rangle h_{I} \otimes h_{J} .
$$

2.4. Averaging over Good Whitney Regions. Let $f \in$ $L^{2}\left(\mathbb{R}^{n+m}\right)$. Let always $I_{1}, I_{2} \in \mathscr{D}_{n}$ and $J_{1}, J_{2} \in \mathscr{D}_{m}$. Note that the position and goodness of $I+\beta_{n}$ are independent. Therefore, one can write

$$
\begin{aligned}
& \left\|g_{\lambda_{1}, \lambda_{2}}^{*}(f)\right\|_{L^{2}\left(\mathbb{R}^{n+m}\right)}^{2}=\iint_{\mathbb{R}_{+}^{m+1}} \iint_{\mathbb{R}_{+}^{n+1}} \iint_{\mathbb{R}^{n+m}}\left|\theta_{t_{1}, t_{2}} f(x-y)\right|^{2}\left(\frac{t_{1}}{t_{1}+\left|y_{1}\right|}\right)^{n \lambda_{1}} \times\left(\frac{t_{2}}{t_{2}+\left|y_{2}\right|}\right)^{m \lambda_{2}} \frac{d y_{1}}{t_{1}^{n}} \frac{d y_{2}}{t_{2}^{m}} \frac{d x_{1} d t_{1}}{t_{1}} \frac{d x_{2} d t_{2}}{t_{2}} \\
& \quad=\frac{1}{\pi_{\text {good }}^{n}} \frac{1}{\pi_{\text {good }}^{m}} \\
& \quad \cdot \mathbb{E}_{\beta_{n}, \beta_{m}} \sum_{I_{2}, J_{2} \text { :good }} \iint_{W_{J_{2}}} \iint_{W_{I_{2}}} \iint_{\mathbb{R}^{n+m}}\left|\theta_{t_{1}, t_{2}} f(x-y)\right|^{2} \times\left(\frac{t_{1}}{t_{1}+\left|y_{1}\right|}\right)^{n \lambda_{1}}\left(\frac{t_{2}}{t_{2}+\left|y_{2}\right|}\right)^{m \lambda_{2}} \frac{d y_{1}}{t_{1}^{n}} \frac{d y_{2}}{t_{2}^{m}} \frac{d x_{1} d t_{1}}{t_{1}} \frac{d x_{2} d t_{2}}{t_{2}}=\frac{1}{\pi_{\text {good }}^{n}} \\
& \quad \frac{1}{\pi_{\text {good }}^{m}} \sum_{I_{I_{2}, J_{2}: \text { good }}} \iint_{W_{J_{2}}} \iint_{W_{I_{2}}} \iint_{\mathbb{R}^{n+m}}\left|\sum_{I_{1}, J_{1}} f_{I_{1} J_{1}} \theta_{t_{1}, t_{2}} h_{I_{1} \times J_{1}}(x-y)\right|^{2} \times\left(\frac{t_{1}}{t_{1}+\left|y_{1}\right|}\right)^{n \lambda_{1}}\left(\frac{t_{2}}{t_{2}+\left|y_{2}\right|}\right)^{m \lambda_{2}} \frac{d y_{1}}{t_{1}^{n}} \frac{d y_{2}}{t_{2}^{m}} \frac{d x_{1}}{t_{1}} \frac{d t_{1}}{\frac{d x_{2}}{t_{2}}} .
\end{aligned}
$$

Indeed, to get this equality, we only need to apply the similar argument to one-parameter case twice. For more details in one-parameter setting, see [5]. Consequently, we are reduced to bound the sum 


$$
\mathscr{G}:=\sum_{I_{2}, J_{2}: \text { good }} \iint_{W_{J_{2}}} \iint_{W_{I_{2}}} \iint_{\mathbb{R}^{n+m}}\left|\sum_{I_{1}, J_{1}} f_{I_{1} J_{1}} \theta_{t_{1}, t_{2}} h_{I_{1} \times J_{1}}(x-y)\right|^{2} \times\left(\frac{t_{1}}{t_{1}+\left|y_{1}\right|}\right)^{n \lambda_{1}}\left(\frac{t_{2}}{t_{2}+\left|y_{2}\right|}\right)^{m \lambda_{2}} \frac{d y_{1}}{t_{1}^{n}} \frac{d y_{2}}{t_{2}^{m}} \frac{d x_{1} d t_{1}}{t_{1}} \frac{d x_{2} d t_{2}}{t_{2}} .
$$

Furthermore, we can carry out the decomposition

$$
\mathscr{G} \leqslant \mathscr{G}_{<,<}+\mathscr{G}_{<, \geq}+\mathscr{G}_{\geq,<}+\mathscr{G}_{\geq, \geq}
$$

$$
\begin{aligned}
& \mathscr{G}_{<,<} \\
& :=\sum_{I_{2}, J_{2}: \text { good }} \iint_{W_{I_{2}}} \iint_{W_{I_{2}}} \iint_{\mathbb{R}^{n+m}}\left|\sum_{\substack{I_{1}, J_{1} \\
\ell\left(I_{1}\right)<\ell\left(I_{2}\right) \\
\ell\left(J_{1}\right)<\ell\left(J_{2}\right)}} f_{I_{1} J_{1}} \theta_{t_{1}, t_{2}} h_{I_{1} \times J_{1}}(x-y)\right|
\end{aligned}
$$

and the others are completely similar.

Sequentially, it is enough to focus on estimating the four pieces: $\mathscr{G}_{<,<}, \mathscr{G}_{<, \geq}, \mathscr{G}_{\geq,<}$, and $\mathscr{G}_{\geq, \geq}$in the following sections.

\section{The Case: $\ell\left(I_{1}\right)<\ell\left(I_{2}\right)$ and $\ell\left(J_{1}\right)<\ell\left(J_{2}\right)$}

For the sake of convenience, we first present two key lemmas, which will be used later.

Lemma 4 (see $[8,14])$. Let

$$
A_{I_{1} I_{2}}=\frac{\ell\left(I_{1}\right)^{\alpha / 2} \ell\left(I_{2}\right)^{\alpha / 2}}{D\left(I_{1}, I_{2}\right)^{n+\alpha}}\left|I_{1}\right|^{1 / 2}\left|I_{2}\right|^{1 / 2}
$$

where the long distance $D\left(I_{1}, I_{2}\right)=\ell\left(I_{1}\right)+\ell\left(I_{2}\right)+d\left(I_{1}, I_{2}\right)$, $I_{1}, I_{2} \in \mathscr{D}_{n}$ and $\alpha>0$. Then for any $x_{I_{1}}, y_{I_{2}} \geq 0$, we have the following estimate:

$$
\left(\sum_{I_{1}, I_{2}} A_{I_{1} I_{2}} x_{I_{1}} y_{I_{2}}\right)^{2} \leqslant \sum_{I_{1}} x_{I_{1}}^{2} \times \sum_{I_{2}} y_{I_{2}}^{2}
$$

In particular, it holds that

$$
\sum_{I_{2}}\left[\sum_{I_{1}} A_{I_{1} I_{2}} x_{I_{1}}\right]^{2} \lesssim \sum_{I_{1}} x_{I_{1}}^{2}
$$

Lemma 5. Let $0<\alpha \leq n\left(\lambda_{1}-2\right) / 2$. For a given cube $I_{2} \in \mathscr{D}_{n}$ and $\left(x_{1}, t_{1}\right) \in W_{I_{2}}$, it holds where

$\times\left(\frac{t_{1}}{t_{1}+\left|y_{1}\right|}\right)^{n \lambda_{1}}\left(\frac{t_{2}}{t_{2}+\left|y_{2}\right|}\right)^{m \lambda_{2}} \frac{d y_{1}}{t_{1}^{n}} \frac{d y_{2}}{t_{2}^{m}} \frac{d x_{1} d t_{1}}{t_{1}} \frac{d x_{2} d t_{2}}{t_{2}}$

$$
\begin{aligned}
& {\left[\int_{\mathbb{R}^{n}}\left(\int_{I_{1}} \frac{d z_{1}}{\left(t_{1}+\left|x_{1}-y_{1}-z_{1}\right|\right)^{n+\alpha}}\right)^{2}\right.} \\
& \left.\cdot\left(\frac{t_{1}}{t_{1}+\left|y_{1}\right|}\right)^{n \lambda_{1}} \frac{d y_{1}}{t_{1}^{n}}\right]^{1 / 2} \\
& \quad \leq \frac{\left|I_{1}\right|}{\left(\ell\left(I_{2}\right)+d\left(I_{1}, I_{2}\right)\right)^{n+\alpha}} .
\end{aligned}
$$

Proof. Fixed $\left(x_{1}, t_{1}\right) \in W_{I_{2}}$. If $\left|y_{1}\right| \leq(1 / 2) d\left(I_{1}, I_{2}\right)$, then

$$
\begin{aligned}
t_{1}+\left|x_{1}-y_{1}-z_{1}\right| & \geq \ell\left(I_{2}\right)+\left|x_{1}-z_{1}\right|-\left|y_{1}\right| \\
& \gtrsim \ell\left(I_{2}\right)+d\left(I_{1}, I_{2}\right) .
\end{aligned}
$$

Thus

$$
\begin{aligned}
& {\left[\int_{\left|y_{1}\right| \leq(1 / 2) d\left(I_{1}, I_{2}\right)}\left(\int_{I_{1}} \frac{d z_{1}}{\left(t_{1}+\left|x_{1}-y_{1}-z_{1}\right|\right)^{n+\alpha}}\right)^{2}\right.} \\
& \left.\cdot\left(\frac{t_{1}}{t_{1}+\left|y_{1}\right|}\right)^{n \lambda_{1}} \frac{d y_{1}}{t_{1}^{n}}\right]^{1 / 2}
\end{aligned}
$$

$$
\lesssim \frac{\left|I_{1}\right|}{\left(\ell\left(I_{2}\right)+d\left(I_{1}, I_{2}\right)\right)^{n+\alpha}}
$$

If $\left|y_{1}\right|>(1 / 2) d\left(I_{1}, I_{2}\right)$, then

$$
\left(\frac{t_{1}}{t_{1}+\left|y_{1}\right|}\right)^{n \lambda_{1}} \frac{1}{t_{1}^{n}} \lesssim \frac{\ell\left(I_{2}\right)^{n \lambda_{1}-n}}{\left(\ell\left(I_{2}\right)+d\left(I_{1}, I_{2}\right)\right)^{n \lambda_{1}}} .
$$


Hence,

$$
\begin{aligned}
& {\left[\int_{\left|y_{1}\right|>(1 / 2) d\left(I_{1}, I_{2}\right)}\left(\int_{I_{1}} \frac{d z_{1}}{\left(t_{1}+\left|x_{1}-y_{1}-z_{1}\right|\right)^{n+\alpha}}\right)^{2}\right.} \\
& \left.\cdot\left(\frac{t_{1}}{t_{1}+\left|y_{1}\right|}\right)^{n \lambda_{1}} \frac{d y_{1}}{t_{1}^{n}}\right]^{1 / 2} \\
& \quad \lesssim \frac{\ell\left(I_{2}\right)^{n \lambda_{1} / 2-n / 2}}{\left(\ell\left(I_{2}\right)+d\left(I_{1}, I_{2}\right)\right)^{n \lambda_{1} / 2}}\left\|\psi_{t_{1}} * \mathbf{1}_{I_{1}}\right\|_{L^{2}\left(\mathbb{R}^{n}\right)} \\
& \quad \leq \frac{\ell\left(I_{2}\right)^{n \lambda_{1} / 2-n / 2}}{\left(\ell\left(I_{2}\right)+d\left(I_{1}, I_{2}\right)\right)^{n \lambda_{1} / 2}}\left\|\psi_{t_{1}}\right\|_{L^{2}\left(\mathbb{R}^{n}\right)}\left|I_{1}\right| \\
& \quad \leq \frac{\ell\left(I_{2}\right)^{n \lambda_{1} / 2-n-\alpha}}{\left(\ell\left(I_{2}\right)+d\left(I_{1}, I_{2}\right)\right)^{n \lambda_{1} / 2}}\left|I_{1}\right| \\
& \quad \leqslant \frac{\left|I_{1}\right|}{\left(\ell\left(I_{2}\right)+d\left(I_{1}, I_{2}\right)\right)^{n+\alpha}},
\end{aligned}
$$

where $\psi_{t_{1}}\left(z_{1}\right)=\left(t_{1}+\left|z_{1}\right|\right)^{-n-\alpha}$ and we have used the condition $0<\alpha \leq n\left(\lambda_{1}-2\right) / 2$ in the last step.

Now we turn our attention to the estimate of $\mathscr{G}_{<,<}$. An easy consequence of the Hölder estimates of the kernel $K_{t_{1}, t_{2}}$ is that

$$
\begin{aligned}
& \left|\theta_{t_{1}, t_{2}} h_{I_{1} \times J_{1}}(x-y)\right| \\
& \leq\left|I_{1}\right|^{-1 / 2} \int_{I_{1}} \frac{\ell\left(I_{1}\right)^{\alpha}}{\left(t_{1}+\left|x_{1}-y_{1}-z_{1}\right|\right)^{n+\alpha}} d z_{1} \\
& \quad \times\left|J_{1}\right|^{-1 / 2} \int_{J_{1}} \frac{\ell\left(J_{1}\right)^{\beta}}{\left(t_{2}+\left|x_{2}-y_{2}-z_{2}\right|\right)^{m+\beta}} d z_{2} .
\end{aligned}
$$

$\mathscr{G}_{<,}$

$$
\begin{aligned}
& \lesssim \sum_{I_{2}, J_{2}: \operatorname{good}} \iint_{W_{J_{2}}} \iint_{W_{I_{2}}}\left[\sum_{\substack{\ell\left(I_{1}\right)<\ell\left(I_{2}\right) \\
\ell\left(J_{1}\right)<\ell\left(J_{2}\right)}}\left|f_{I_{1} J_{1}}\right|\left(\iint_{\mathbb{R}^{n+m}}\left|\theta_{t_{1}, t_{2}} h_{I_{1} \times J_{1}}(x-y)\right|^{2} \times\left(\frac{t_{1}}{t_{1}+\left|y_{1}\right|}\right)^{n \lambda_{1}}\left(\frac{t_{2}}{t_{2}+\left|y_{2}\right|}\right)^{m \lambda_{2}} \frac{d y_{1}}{t_{1}^{n}} \frac{d y_{2}}{t_{2}^{m}}\right)^{1 / 2}\right]^{2} \frac{d x_{1} d t_{1}}{t_{1}} \frac{d x_{2} d t_{2}}{t_{2}} \\
& \lesssim \sum_{J_{2}} \sum_{I_{2}}\left[\sum_{I_{1}} A_{I_{1} I_{2}} \sum_{J_{1}} A_{J_{1} J_{2}}\left|f_{I_{1} J_{1}}\right|\right]^{2} \lesssim \sum_{J_{2}} \sum_{I_{1}}\left[\sum_{J_{1}} A_{J_{1} J_{2}}\left|f_{I_{1} J_{1}}\right|\right]^{2} \lesssim \sum_{I_{1}} \sum_{J_{1}}\left|f_{I_{1} J_{1}}\right|^{2}=\|f\|_{L^{2}\left(\mathbb{R}^{n+m}\right)}^{2} .
\end{aligned}
$$

Therefore, from Minkowski's integral inequality and Lemma 4, it now follows that

Since $\ell\left(I_{1}\right)<\ell\left(I_{2}\right)$ and $\ell\left(J_{1}\right)<\ell\left(J_{2}\right)$, then we get

$$
\mathscr{P}(x, t) \lesssim A_{I_{1} I_{2}}\left|I_{2}\right|^{-1 / 2} \cdot A_{J_{1} J_{2}}\left|J_{2}\right|^{-1 / 2} .
$$

$$
\begin{aligned}
\mathscr{P} & (x, t):=\left(\iint_{\mathbb{R}^{n+m}}\left|\theta_{t_{1}, t_{2}} h_{I_{1} \times J_{1}}(x-y)\right|^{2}\right. \\
\cdot & \left.\left(\frac{t_{1}}{t_{1}+\left|y_{1}\right|}\right)^{n \lambda_{1}}\left(\frac{t_{2}}{t_{2}+\left|y_{2}\right|}\right)^{m \lambda_{2}} \frac{d y_{1}}{t_{1}^{n}} \frac{d y_{2}}{t_{2}^{m}}\right)^{1 / 2} \\
& \leqslant\left|I_{1}\right|^{-1 / 2}\left[\int_{\mathbb{R}^{n}}\left(\int_{I_{1}} \frac{\left.\ell\left(I_{1}\right)^{\alpha} d z_{1}+\left|x_{1}-y_{1}-z_{1}\right|\right)^{n+\alpha}}{t_{1}}\right)^{2}\right. \\
& \left.\cdot\left(\frac{t_{1}}{t_{1}+\left|y_{1}\right|}\right)^{n \lambda_{1}} \frac{d y_{1}}{t_{1}^{n}}\right]^{1 / 2} \times\left|J_{1}\right|^{-1 / 2} \\
& \cdot\left[\int_{\mathbb{R}^{m}}\left(\int_{J_{1}} \frac{\ell\left(t_{2}+\left|x_{2}-y_{2}-z_{2}\right|\right)^{m+\beta}}{\left(z_{2}\right.}\right)^{2}\right. \\
& \left.\cdot \frac{t_{2}}{\left(\ell\left(J_{2}\right)+d\left(J_{1}, J_{2}\right)\right)^{m+\beta}}\left|J_{1}\right|^{1 / 2} \cdot \frac{d y_{2}}{t_{2}^{n}}\right]^{1 / 2} \\
& \leqslant\left(y_{2} \mid\right. \\
& \left(\ell\left(I_{2}\right)+d\left(I_{1}, I_{2}\right)\right)^{n+\alpha}\left|I_{1}\right|^{1 / 2}
\end{aligned}
$$

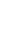

4. The Case: $\ell\left(I_{1}\right) \geq \ell\left(I_{2}\right)$ and $\ell\left(J_{1}\right)<\ell\left(J_{2}\right)$

In any case, we perform the splitting

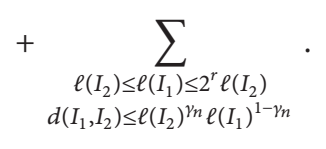

These three parts are called separated, Nested, and adjacent, respectively. The term Nested makes sense, since the 
summing conditions that $I_{2}$ is good actually imply that $I_{1}$ is where the ancestor of $I_{2}$. Thus, it holds

$$
\mathscr{G}_{\geq,<} \lesssim \mathscr{G}_{\text {sep },<}+\mathscr{G}_{\mathrm{nes},<}+\mathscr{G}_{\mathrm{adj},<}
$$

$$
\begin{aligned}
& \mathscr{G}_{\text {sep },<} \\
& =\sum_{I_{2}, J_{2}: \text { good }} \iint_{W_{I_{2}}} \iint_{W_{I_{2}}} \iint_{\mathbb{R}^{n+m}}\left|\sum_{\substack{I_{1}: \ell\left(I_{1}\right) \geq \ell\left(I_{2}\right) \\
d\left(I_{1}, I_{2}\right)>\ell\left(I_{2}\right)^{\gamma_{n}} \ell\left(I_{1}\right)^{1-\gamma_{n}}}} \sum_{J_{1}: \ell\left(J_{1}\right)<\ell\left(J_{2}\right)} f_{I_{1} I_{1}} \times \theta_{t_{1}, t_{2}} h_{I_{1} \times J_{1}}(x-y)\right|^{2}\left(\frac{t_{1}}{t_{1}+\left|y_{1}\right|}\right)^{n \lambda_{1}}\left(\frac{t_{2}}{t_{2}+\left|y_{2}\right|}\right)^{m \lambda_{2}} \frac{d y_{1}}{t_{1}^{n}} \frac{d y_{2}}{t_{2}^{m}} \frac{d x_{1} d t_{1}}{t_{1}} \frac{d x_{2} d t_{2}}{t_{2}}, \\
& \mathscr{G}_{\text {nes, }<} \\
& =\sum_{I_{2}, J_{2}: \text { good }} \iint_{W_{I_{2}}} \iint_{W_{I_{2}}} \iint_{\mathbb{R}^{n+m}}\left|\sum_{\substack{I_{1}: \ell\left(I_{1}\right)>2^{r} \ell\left(I_{2}\right) \\
d\left(I_{1}, I_{2}\right) \leq \ell\left(I_{2}\right)^{\gamma_{n}} \ell\left(I_{1}\right)^{1-\gamma_{n}}}} \sum_{J_{1}: \ell\left(J_{1}\right)<\ell\left(J_{2}\right)} f_{I_{1} J_{1}} \times \theta_{t_{1}, t_{2}} h_{I_{1} \times J_{1}}(x-y)\right|^{2}\left(\frac{t_{1}}{t_{1}+\left|y_{1}\right|}\right)^{n \lambda_{1}}\left(\frac{t_{2}}{t_{2}+\left|y_{2}\right|}\right)^{m \lambda_{2}} \frac{d y_{1}}{t_{1}^{n}} \frac{d y_{2}}{t_{2}^{m}} \frac{d x_{1} d t_{1}}{t_{1}} \frac{d x_{2} d t_{2}}{t_{2}}, \\
& \mathscr{G}_{\text {adj },<} \\
& =\sum_{I_{2}, J_{2}: \text { good }} \iint_{W_{J_{2}}} \iint_{W_{I_{2}}} \iint_{\mathbb{R}^{n+m}}\left|\sum_{\substack{I_{1}: \ell\left(I_{2}\right) \leq \ell\left(I_{1}\right) \leq 2^{r} \ell\left(I_{2}\right) \\
d\left(I_{1}, I_{2}\right) \leq \ell\left(I_{2}\right)^{\gamma_{n}} \ell\left(I_{1}\right)^{1-\gamma_{n}}}} \sum_{J_{1}: \ell\left(J_{1}\right)<\ell\left(J_{2}\right)} f_{I_{1} J_{1}} \times \theta_{t_{1}, t_{2}} h_{I_{1} \times J_{1}}(x-y)\right|^{2}\left(\frac{t_{1}}{t_{1}+\left|y_{1}\right|}\right)^{n \lambda_{1}}\left(\frac{t_{2}}{t_{2}+\left|y_{2}\right|}\right)^{m \lambda_{2}} \frac{d y_{1}}{t_{1}^{n}} \frac{d y_{2}}{t_{2}^{m}} \frac{d x_{1} d t_{1}}{t_{1}} \frac{d x_{2} d t_{2}}{t_{2}} .
\end{aligned}
$$

Now we are in position to estimate the above three terms, respectively.

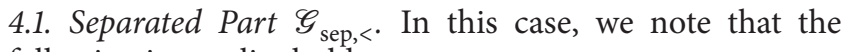
following inequality holds:

$$
\frac{t_{1}^{\alpha}}{\left(\ell\left(I_{2}\right)+d\left(I_{1}, I_{2}\right)\right)^{n+\alpha}}\left|I_{1}\right|^{1 / 2} \lesssim A_{I_{1} I_{2}}\left|I_{2}\right|^{-1 / 2} .
$$

Indeed, if $d\left(I_{1}, I_{2}\right) \geq \ell\left(I_{1}\right)$, then $D\left(I_{1}, I_{2}\right) \sim d\left(I_{1}, I_{2}\right)$. Therefore, we get

$$
\frac{t_{1}^{\alpha}}{\left(\ell\left(I_{2}\right)+d\left(I_{1}, I_{2}\right)\right)^{n+\alpha}}\left|I_{1}\right|^{1 / 2} \lesssim A_{I_{1} I_{2}}\left|I_{2}\right|^{-1 / 2} .
$$

If $d\left(I_{1}, I_{2}\right)<\ell\left(I_{1}\right)$, then $D\left(I_{1}, I_{2}\right) \sim \ell\left(I_{1}\right)$. Moreover, noticing that $\gamma_{n}(n+\alpha)=\alpha / 2$ and $d\left(I_{1}, I_{2}\right)>\ell\left(I_{2}\right)^{\gamma_{n}} \ell\left(I_{1}\right)^{1-\gamma_{n}}$, one may conclude that

$$
\begin{aligned}
& \frac{t_{1}^{\alpha}}{\left(\ell\left(I_{2}\right)+d\left(I_{1}, I_{2}\right)\right)^{n+\alpha}}\left|I_{1}\right|^{1 / 2} \\
& \lesssim \frac{\ell\left(I_{1}\right)^{\alpha / 2} \ell\left(I_{2}\right)^{\alpha / 2}}{\ell\left(I_{1}\right)^{n+\alpha}}\left|I_{1}\right|^{1 / 2} \lesssim A_{I_{1} I_{2}}\left|I_{2}\right|^{-1 / 2} .
\end{aligned}
$$

It is obvious that the mixed Hölder and size condition implies that

$$
\begin{aligned}
& \left|\theta_{t_{1}, t_{2}} h_{I_{1} \times J_{1}}(x-y)\right| \\
& \leq\left|I_{1}\right|^{-1 / 2} \int_{I_{1}} \frac{t_{1}^{\alpha}}{\left(t_{1}+\left|x_{1}-y_{1}-z_{1}\right|\right)^{n+\alpha}} d z_{1} \\
& \quad \times\left|J_{1}\right|^{-1 / 2} \int_{J_{1}} \frac{\ell\left(J_{1}\right)^{\beta}}{\left(t_{2}+\left|x_{2}-y_{2}-z_{2}\right|\right)^{m+\beta}} d z_{2} .
\end{aligned}
$$

Thus, combining Lemma 5 with (39), one can obtain

$$
\begin{aligned}
\mathscr{P} & (x, t) \lesssim\left|I_{1}\right|^{-1 / 2} \\
\cdot & {\left[\int_{\mathbb{R}^{n}}\left(\int_{I_{1}} \frac{t_{1}^{\alpha} d z_{1}}{\left(t_{1}+\left|x_{1}-y_{1}-z_{1}\right|\right)^{n+\alpha}}\right)^{2}\right.} \\
\cdot & \left.\left(\frac{t_{1}}{t_{1}+\left|y_{1}\right|}\right)^{n \lambda_{1}} \frac{d y_{1}}{t_{1}^{n}}\right]^{1 / 2} \times\left|J_{1}\right|^{-1 / 2} \\
& {\left[\int_{\mathbb{R}^{m}}\left(\int_{J_{1}} \frac{\ell\left(J_{1}\right)^{\beta} d z_{2}}{\left(t_{2}+\left|x_{2}-y_{2}-z_{2}\right|\right)^{m+\beta}}\right)^{2}\right.} \\
& \left.\left(\frac{t_{2}}{t_{2}+\left|y_{2}\right|}\right)^{m \lambda_{2}} \frac{d y_{2}}{t_{2}^{n}}\right]^{1 / 2}
\end{aligned}
$$




$$
\begin{aligned}
& \lesssim \frac{t_{1}^{\alpha}}{\left(\ell\left(I_{2}\right)+d\left(I_{1}, I_{2}\right)\right)^{n+\alpha}}\left|I_{1}\right|^{1 / 2} \\
& \cdot \frac{\ell\left(J_{1}\right)^{\beta}}{\left(\ell\left(J_{2}\right)+d\left(J_{1}, J_{2}\right)\right)^{m+\beta}}\left|J_{1}\right|^{1 / 2} \\
& \lesssim \frac{\ell\left(I_{2}\right)^{\alpha}}{d\left(I_{1}, I_{2}\right)^{n+\alpha}\left|I_{1}\right|^{1 / 2} A_{J_{1} J_{2}}\left|J_{2}\right|^{-1 / 2} \lesssim A_{I_{1} I_{2}}\left|I_{2}\right|^{-1 / 2}} \\
& \cdot A_{J_{1} J_{2}}\left|J_{2}\right|^{-1 / 2} .
\end{aligned}
$$

Consequently, by the similar argument as $\mathscr{G}_{<,<}$, we have

$$
\mathscr{G}_{\text {sep },<} \lesssim\|f\|_{L^{2}\left(\mathbb{R}^{n+m}\right)}^{2} .
$$

4.2. Adjacent Part $\mathscr{G}_{\mathrm{adj},<\cdot}$ The summation conditions $\ell\left(I_{2}\right) \leq$ $\ell\left(I_{1}\right) \leq 2^{r} \ell\left(I_{2}\right)$ and $d\left(I_{1}, I_{2}\right) \leq \ell\left(I_{2}\right)^{\gamma_{n}} \ell\left(I_{1}\right)^{1-\gamma_{n}}$ indicate that $\ell\left(I_{1}\right) \sim \ell\left(I_{2}\right) \sim D\left(I_{1}, I_{2}\right)$. Thus,

$$
\frac{\ell\left(I_{2}\right)^{\alpha}}{\left(\ell\left(I_{2}\right)+d\left(I_{1}, I_{2}\right)\right)^{n+\alpha}}\left|I_{1}\right|^{1 / 2} \lesssim \ell\left(I_{2}\right)^{-n}
$$

$$
\sim \frac{\ell\left(I_{1}\right)^{\alpha / 2} \ell\left(I_{2}\right)^{\alpha / 2}}{D\left(I_{1}, I_{2}\right)^{n+\alpha}} .
$$

It follows from (43) that

$$
\begin{aligned}
\mathscr{P}(x, t) \lesssim & \frac{\ell\left(I_{2}\right)^{\alpha}}{\left(\ell\left(I_{2}\right)+d\left(I_{1}, I_{2}\right)\right)^{n+\alpha}}\left|I_{1}\right|^{1 / 2} \\
& \cdot A_{J_{1} J_{2}}\left|J_{2}\right|^{-1 / 2} \\
\lesssim & A_{I_{1} I_{2}}\left|I_{2}\right|^{-1 / 2} \cdot A_{J_{1} J_{2}}\left|J_{2}\right|^{-1 / 2} .
\end{aligned}
$$

Therefore, exactly as we have seen before,

$$
\mathscr{G}_{\mathrm{adj},<} \lesssim\|f\|_{L^{2}\left(\mathbb{R}^{n+m}\right)}^{2} .
$$

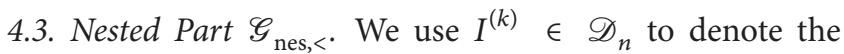
unique cube for which $\ell\left(I^{(k)}\right)=2^{k} \ell(I)$ and $I \subset I^{(k)}$. We call $I^{(k)}$ as the $k$ generation older dyadic ancestor of $I$. In this case, by the goodness of $I_{2}$, it must actually have $I_{2} \subsetneq I_{1}$. That is, $I_{1}$ is the ancestor of $I_{2}$. This enables us to write

$$
\begin{aligned}
& \mathscr{G}_{\text {nes, }<} \\
& =\sum_{I, J_{2}: \text { good }} \iint_{W_{J_{2}}} \iint_{W_{I}} \iint_{\mathbb{R}^{n+m}}\left|\sum_{k=1}^{\infty} \sum_{J_{1}: \ell\left(J_{1}\right)<\ell\left(J_{2}\right)} f_{I^{(k)} J_{1}} \theta_{t_{1}, t_{2}} h_{I^{(k)} \times J_{1}}(x-y)\right|^{2} \times\left(\frac{t_{1}}{t_{1}+\left|y_{1}\right|}\right)^{n \lambda_{1}}\left(\frac{t_{2}}{t_{2}+\left|y_{2}\right|}\right)^{m \lambda_{2}} \frac{d y_{1}}{t_{1}^{n}} \frac{d y_{2}}{t_{2}^{m}} \frac{d x_{1} d t_{1}}{t_{1}} \frac{d x_{2} d t_{2}}{t_{2}} .
\end{aligned}
$$

Introduce the notation

$$
s_{I}^{k}=-\mathbf{1}_{\left(I^{(k-1)}\right)^{c}}\left\langle h_{I^{(k)}}\right\rangle_{I^{(k-1)}}+\sum_{\substack{I^{\prime} \in \operatorname{ch}\left(I^{(k)}\right) \\ I^{\prime} \neq I^{(k-1)}}} \mathbf{1}_{I^{\prime}} h_{I^{(k)}} .
$$

Then, it is easy to check that

$$
h_{I^{(k)}}=s_{I}^{k}+\left\langle h_{I^{(k)}}\right\rangle_{I^{(k-1)}},
$$

$\operatorname{supp} s_{I}^{k} \subset\left(I^{(k-1)}\right)^{c}$, and $\left|s_{I}^{k}\right| \lesssim\left|I^{(k)}\right|^{-1 / 2}$.
Denote $f_{J_{1}}=\left\langle f, h_{J_{1}}\right\rangle$ so that $f_{J_{1}}=\int_{\mathbb{R}^{m}} f\left(y_{1}, y_{2}\right) d y_{2}$, $y_{1} \in \mathbb{R}^{n}$. Then, we split

$$
\mathscr{G}_{\text {nes },<} \lesssim \mathscr{G}_{\text {mod, }<}+\mathscr{G}_{\mathrm{Car},<}
$$

where

$$
\begin{aligned}
& \mathscr{G}_{\text {mod, }<}=\sum_{I, J_{2}: \text { good }} \iint_{W_{J_{2}}} \iint_{W_{I}} \iint_{\mathbb{R}^{n+m}}\left|\sum_{k=1}^{\infty} \sum_{J_{1}: \ell\left(J_{1}\right)<\ell\left(J_{2}\right)} f_{I^{(k)} J_{1}} \theta_{t_{1}, t_{2}}\left(s_{I}^{k} \otimes h_{J_{1}}\right)(x-y)\right|^{2} \times\left(\frac{t_{1}}{t_{1}+\left|y_{1}\right|}\right)^{n \lambda_{1}}\left(\frac{t_{2}}{t_{2}+\left|y_{2}\right|}\right)^{m \lambda_{2}} \frac{d y_{1}}{t_{1}^{n}} \frac{d y_{2}}{t_{2}^{m}} \frac{d x_{1} d t_{1}}{t_{1}} \frac{d x_{2} d t_{2}}{t_{2}}, \\
& \mathscr{G}_{\mathrm{Car},<} \\
& =\sum_{I, J_{2}: \text { good }} \iint_{W_{J_{2}}} \iint_{W_{I}} \iint_{\mathbb{R}^{n+m}}\left|\sum_{J_{1}: \ell\left(J_{1}\right)<\ell\left(J_{2}\right)} f_{I^{(k)} J_{1}} \theta_{t_{1}, t_{2}}\left(\mathbf{1} \otimes h_{J_{1}}\right)(x-y) \times \sum_{k=1}^{\infty}\left\langle\Delta_{I^{(k)}} f_{J_{1}}\right\rangle_{I^{(k-1)}}\right|^{2}\left(\frac{t_{1}}{t_{1}+\left|y_{1}\right|}\right)^{n \lambda_{1}}\left(\frac{t_{2}}{t_{2}+\left|y_{2}\right|}\right)^{m \lambda_{2}} \frac{d y_{1}}{t_{1}^{n}} \frac{d y_{2}}{t_{2}^{m}} \frac{d x_{1} d t_{1}}{t_{1}} \frac{d x_{2} d t_{2}}{t_{2}} \\
& =\sum_{I, J_{2}: \text { good }} \iint_{W_{I_{2}}} \iint_{W_{I}} \iint_{\mathbb{R}^{n+m}}\left|\sum_{J_{1}: \ell\left(J_{1}\right)<\ell\left(J_{2}\right)}\left\langle f_{J_{1}}\right\rangle_{I} \theta_{t_{1}, t_{2}}\left(\mathbf{1} \otimes h_{J_{1}}\right)(x-y)\right|^{2} \times\left(\frac{t_{1}}{t_{1}+\left|y_{1}\right|}\right)^{n \lambda_{1}}\left(\frac{t_{2}}{t_{2}+\left|y_{2}\right|}\right)^{m \lambda_{2}} \frac{d y_{1}}{t_{1}^{n}} \frac{d y_{2}}{t_{2}^{m}} \frac{d x_{1} d t_{1}}{t_{1}} \frac{d x_{2} d t_{2}}{t_{2}} .
\end{aligned}
$$


4.3.1. Estimate of $\mathscr{G}_{\bmod ,<\cdot}$ We need the following lemma.

Lemma 6. Let $0<\beta \leq m\left(\lambda_{2}-2\right) / 2$ and $k \in \mathbb{N}_{+}$. Given cubes $I \in \mathscr{D}_{n}, J_{1}, J_{2} \in \mathscr{D}_{m},\left(x_{1}, t_{1}\right) \in W_{I}$, and $\left(x_{2}, t_{2}\right) \in W_{J_{1}}$, the following estimate holds:

$$
\begin{aligned}
& \mathbb{Q}(x, t):=\left(\iint_{\mathbb{R}^{n+m}}\left|\theta_{t_{1}, t_{2}}\left(s_{I}^{k} \otimes h_{J_{1}}\right)(x-y)\right|^{2}\right. \\
& \left.\cdot\left(\frac{t_{1}}{t_{1}+\left|y_{1}\right|}\right)^{n \lambda_{1}}\left(\frac{t_{2}}{t_{2}+\left|y_{2}\right|}\right)^{m \lambda_{2}} \frac{d y_{1}}{t_{1}^{n}} \frac{d y_{2}}{t_{2}^{m}}\right)^{1 / 2} \\
& \quad \leq 2^{-\alpha k / 2}\left|I^{(k)}\right|^{-1 / 2}\left|J_{1}\right|^{1 / 2} \frac{\ell\left(J_{1}\right)^{\beta}}{\left(\ell\left(J_{2}\right)+d\left(J_{1}, J_{2}\right)\right)^{m+\beta}} .
\end{aligned}
$$

$$
\mathscr{K}:=\left[\int_{\mathbb{R}^{n}}\left(\int_{{\left(I^{(k-1)}\right)^{c}}^{c}} \frac{t_{1}^{\alpha} d z_{1}}{\left(t_{1}+\left|x_{1}-y_{1}-z_{1}\right|\right)^{n+\alpha}}\right)^{2}\left(\frac{t_{1}}{t_{1}+\left|y_{1}\right|}\right)^{n \lambda_{1}} \frac{d y_{1}}{t_{1}^{n}}\right]^{1 / 2} \leqslant 2^{-\alpha k / 2} .
$$

Indeed, if $k \leq r$,

$$
\begin{aligned}
\mathscr{K} & \leq\left[\int_{\mathbb{R}^{n}}\left(\int_{\mathbb{R}^{n}} \frac{\ell(I)^{\alpha} d z_{1}}{\left(\ell(I)+\left|x_{1}-y_{1}-z_{1}\right|\right)^{n+\alpha}}\right)^{2}\right. \\
& \left.\cdot\left(\frac{t_{1}}{t_{1}+\left|y_{1}\right|}\right)^{n \lambda_{1}} \frac{d y_{1}}{t_{1}^{n}}\right]^{1 / 2} \\
& \leq \int_{\mathbb{R}^{n}} \frac{\ell(I)^{\alpha}}{\left(\ell(I)+\left|x_{1}-z_{1}\right|\right)^{n+\alpha}} d z_{1} \lesssim \ell(I)^{-n}|I| \\
& +\ell(I)^{\alpha} \int_{I^{c}} \frac{d z_{1}}{\left|z_{1}-x_{1}\right|^{n+\alpha}} \leqslant 1 \sim 2^{-\alpha k / 2} .
\end{aligned}
$$

If $k>r$, we have by the goodness of $I$ that

$$
\begin{aligned}
d\left(I,\left(I^{(k-1)}\right)^{c}\right) & >\ell(I)^{\gamma_{n}} \ell\left(I^{(k-1)}\right)^{1-\gamma_{n}} \\
& =2^{(k-1)\left(1-\gamma_{n}\right)} \ell(I) \geq 2^{k / 2} \ell(I) .
\end{aligned}
$$

Thus, we obtain

$$
\begin{aligned}
& \int_{\left(I^{(k-1)}\right)^{c}} \frac{\ell(I)^{\alpha}}{\left|z_{1}-x_{1}\right|^{n+\alpha}} d z_{1} \\
& \quad \leq \int_{B\left(x_{1}, d\left(I,\left(I^{(k-1)}\right)^{c}\right)\right)} \frac{\ell(I)^{\alpha}}{\left|z_{1}-x_{1}\right|^{n+\alpha}} d z_{1} \\
& \quad \leq \ell(I)^{\alpha} d\left(I,\left(I^{(k-1)}\right)^{c}\right)^{-\alpha} \leqslant 2^{-\alpha k / 2} .
\end{aligned}
$$

Given $y_{1} \in \mathbb{R}^{n}$, we introduce the notation

$$
\begin{aligned}
& E_{1}=\left\{z_{1} \in\left(I^{(k-1)}\right)^{c} ;\left|z_{1}-x_{1}\right| \geq 2\left|y_{1}\right|\right\}, \\
& E_{2}=\left\{z_{1} \in\left(I^{(k-1)}\right)^{c} ;\left|z_{1}-x_{1}\right|<2\left|y_{1}\right|\right\} .
\end{aligned}
$$

Proof. By the mixed Hölder and size condition, it yields that

$$
\begin{aligned}
& \left|\theta_{t_{1}, t_{2}}\left(s_{I}^{k} \otimes h_{J_{1}}\right)(x-y)\right| \\
& \quad \leq\left|I^{(k)}\right|^{-1 / 2} \int_{\left(I^{(k-1)}\right)^{c}} \frac{t_{1}^{\alpha}}{\left(t_{1}+\left|x_{1}-y_{1}-z_{1}\right|\right)^{n+\alpha}} d z_{1} \\
& \quad \times\left|J_{1}\right|^{-1 / 2} \int_{J_{1}} \frac{\ell\left(J_{1}\right)^{\beta}}{\left(t_{2}+\left|x_{2}-y_{2}-z_{2}\right|\right)^{m+\beta}} d z_{2} .
\end{aligned}
$$

Proceeding as we did in (33), we only need to show
Then, it follows that

$$
\begin{aligned}
\mathscr{K} & \leq\left[\int_{\mathbb{R}^{n}}\left(\int_{E_{1}} \frac{\ell(I)^{\alpha} d z_{1}}{\left(\ell(I)+\left|x_{1}-y_{1}-z_{1}\right|\right)^{n+\alpha}}\right)^{2}\right. \\
& \left.\cdot\left(\frac{t_{1}}{t_{1}+\left|y_{1}\right|}\right)^{n \lambda_{1}} \frac{d y_{1}}{t_{1}^{n}}\right]^{1 / 2} \\
& +\left[\int_{\mathbb{R}^{n}}\left(\int_{E_{2}} \frac{\ell(I)^{\alpha} d z_{1}}{\left(\ell(I)+\left|x_{1}-y_{1}-z_{1}\right|\right)^{n+\alpha}}\right)^{2}\right. \\
& \left.\cdot\left(\frac{t_{1}}{t_{1}+\left|y_{1}\right|}\right)^{n \lambda_{1}} \frac{d y_{1}}{t_{1}^{n}}\right]^{1 / 2}:=\mathscr{K}_{1}+\mathscr{K}_{2} .
\end{aligned}
$$

Note that

$$
\ell(I)+\left|x_{1}-y_{1}-z_{1}\right|>\left|z_{1}-x_{1}\right|-\left|y_{1}\right| \geq \frac{1}{2}\left|z_{1}-x_{1}\right|,
$$$$
\text { whenever } z_{1} \in E_{1} \text {. }
$$

This yields that

$$
\begin{aligned}
\mathscr{K}_{1} \lesssim & \int_{\left(I^{(k-1)}\right)^{c}} \frac{\ell(I)^{\alpha}}{\left|z_{1}-x_{1}\right|^{n+\alpha}} d z_{1} \\
& \cdot\left[\int_{\mathbb{R}^{n}}\left(\frac{t_{1}}{t_{1}+\left|y_{1}\right|}\right)^{n \lambda_{1}} \frac{d y_{1}}{t_{1}^{n}}\right]^{1 / 2} \leq 2^{-\alpha k / 2} .
\end{aligned}
$$

As for $\mathscr{K}_{2}$, we by Young's inequality have the following estimate: 


$$
\begin{aligned}
\mathscr{K}_{2} & \leq \ell(I)^{\alpha}\left[\int_{\mathbb{R}^{n}}\left(\int_{E_{2}} \frac{\ell(I)^{n \lambda_{1} / 2-n / 2}}{\left(\ell(I)+\left|x_{1}-z_{1}\right|\right)^{n \lambda_{1} / 2}} \frac{1}{\left(t_{1}+\left|y_{1}-z_{1}\right|\right)^{n+\alpha}} d z_{1}\right)^{2} d y_{1}\right]^{1 / 2} \\
& \leq \ell(I)^{\alpha}\left[\int_{\mathbb{R}^{n}}\left(\int_{E_{2}} \frac{\ell(I)^{n / 2+\alpha}}{\left(\ell(I)+\left|x_{1}-z_{1}\right|\right)^{n+\alpha}} \frac{1}{\left(t_{1}+\left|y_{1}-z_{1}\right|\right)^{n+\alpha}} d z_{1}\right)^{2} d y_{1}\right]^{1 / 2}=\ell(I)^{n / 2+2 \alpha}\|\xi * \eta\|_{L^{2}\left(\mathbb{R}^{n}\right)} \\
& \leq \ell(I)^{n / 2+2 \alpha}\|\xi\|_{L^{2}\left(\mathbb{R}^{n}\right)}\|\eta\|_{L^{1}\left(\mathbb{R}^{n}\right)} \leqslant \ell(I)^{\alpha} \int_{\left(I^{(k-1)}\right)} \frac{\ell(I)^{\alpha}}{\left|z_{1}-x_{1}\right|^{n+\alpha}} d z_{1} \leq 2^{-\alpha k / 2},
\end{aligned}
$$

where

$$
\eta\left(z_{1}\right)=\frac{\mathbf{1}_{E_{2}}\left(z_{1}\right)}{t_{1}+\left|z_{1}-x_{1}\right|^{n+\alpha}} .
$$

$$
\xi\left(z_{1}\right)=\frac{1}{t_{1}+\left|z_{1}\right|^{n+\alpha}}
$$

Accordingly, Minkowski's integral inequality and Lemma 6 give that

$$
\begin{aligned}
& \mathscr{G}_{\text {mod, }} \\
& \lesssim \sum_{I, J_{2}: \text { :good }} \iint_{W_{J_{2}}} \iint_{W_{I}}\left[\sum_{k=1}^{\infty} \sum_{J_{1}: \ell\left(J_{1}\right)<\ell\left(J_{2}\right)}\left|f_{I^{(k)} J_{1}}\right|\left(\iint_{\mathbb{R}^{n+m}}\left|\theta_{t_{1}, t_{2}}\left(s_{I}^{k} \otimes h_{J_{1}}\right)(x-y)\right|^{2} \times\left(\frac{t_{1}}{t_{1}+\left|y_{1}\right|}\right)^{n \lambda_{1}}\left(\frac{t_{2}}{t_{2}+\left|y_{2}\right|}\right)^{m \lambda_{2}} \frac{d y_{1}}{t_{1}^{n}} \frac{d y_{2}}{t_{2}^{m}}\right)^{1 / 2}\right]^{2} \frac{d x_{1} d t_{1}}{t_{1}} \frac{d x_{2} d t_{2}}{t_{2}} \\
& \leq \sum_{I} \sum_{J_{2}} \iint_{W_{I_{2}}} \iint_{W_{I}}\left[\sum_{k=1}^{\infty} 2^{-\alpha k / 2} \sum_{J_{1} \ell\left(J_{1}\right)<\ell\left(J_{2}\right)} A_{J_{1} I_{2}}\left|f_{I^{(k)} J_{1}}\right|\left|I^{(k)}\right|^{-1 / 2}\left|J_{1}\right|^{1 / 2}\right]^{2} \frac{d x_{1} d t_{1}}{t_{1}} \frac{d x_{2} d t_{2}}{t_{2}} \\
& \leq \sum_{I} \sum_{J_{2}}\left[\sum_{k=1}^{\infty} 2^{-\alpha k / 2}\left(\left.\frac{|I|}{\mid I^{(k)}}\right|_{J_{1}: \ell\left(J_{1}\right)<\ell\left(J_{2}\right)} A_{J_{1} J_{2}}\left|f_{I^{(k)} J_{1}}\right|\right)^{1 / 2}\right]^{2} \leq\left[\sum_{k=1}^{\infty} 2^{-\alpha k / 4} \cdot 2^{-\alpha k / 4}\left(\sum_{I} \frac{|I|}{\left|I^{(k)}\right|} \sum_{J_{2}}\left(\sum_{\left.\left.J_{1}: \ell J_{1}\right)<\ell J_{2}\right)} A_{J_{1} J_{2}}\left|f_{I^{(k)} I_{1}}\right|\right)^{2}\right)^{1 / 2}\right]^{2} \\
& \leqslant \sum_{k=1}^{\infty} 2^{-\alpha k / 2} \sum_{I} \frac{|I|}{\left|I^{(k)}\right|} \sum_{J_{2}}\left(\sum_{J_{1}: \ell\left(J_{1}\right)<\ell\left(J_{2}\right)} A_{J_{1} J_{2}}\left|f_{I^{(k)} J_{1}}\right|\right)^{2} \leqslant \sum_{k=1}^{\infty} 2^{-\alpha k / 2} \sum_{Q, J_{1}} \frac{\left|f_{\mathrm{QI}_{1}}\right|^{2}}{|\mathrm{Q}|} \sum_{I: I^{(k)=Q}}|I| \lesssim\|f\|_{L^{2}\left(\mathbb{R}^{n+m}\right)}^{2} .
\end{aligned}
$$

4.3.2. Estimate of $\mathscr{G}_{\mathrm{Car},<}$. We also need to use the following lemma.

Lemma 7. Let $J_{1}, J_{2} \in \mathscr{D}_{m}$ be cubes, and $\left(x_{2}, t_{2}\right) \in W_{J_{2}}$. Then the Carleson condition holds

$$
\begin{aligned}
& \mathscr{R}\left(x_{2}, t_{2}\right):=\sum_{I^{\prime} \subset I} \iint_{W_{I^{\prime}}} \iint_{\mathbb{R}^{n+m}}\left|\theta_{t_{1}, t_{2}}\left(1 \otimes h_{J_{1}}\right)(x-y)\right|^{2} \\
& \cdot \prod_{i=1}^{2}\left(\frac{t_{i}}{t_{i}+\left|y_{i}\right|}\right)^{n \lambda_{i}} \frac{d y_{1}}{t_{1}^{n}} \frac{d y_{2}}{t_{2}^{m}} \frac{d x_{1} d t_{1}}{t_{1}} \\
& \quad \leq|I|\left(\frac{\ell\left(J_{1}\right)^{\beta}\left|J_{1}\right|^{1 / 2}}{\left(\ell\left(J_{2}\right)+d\left(J_{1}, J_{2}\right)\right)^{m+\beta}}\right)^{2} .
\end{aligned}
$$

Proof. The first step is to split

$$
\mathscr{R}\left(x_{2}, t_{2}\right) \lesssim \mathscr{R}_{1}\left(x_{2}, t_{2}\right)+\mathscr{R}_{2}\left(x_{2}, t_{2}\right),
$$

where

$$
\begin{gathered}
\mathscr{R}_{1}\left(x_{2}, t_{2}\right)=\iint_{\widehat{3 I}} \iint_{\mathbb{R}^{n+m}}\left|\theta_{t_{1}, t_{2}}\left(\mathbf{1}_{3 I} \otimes h_{J_{1}}\right)(x-y)\right|^{2} \\
\cdot \prod_{i=1}^{2}\left(\frac{t_{i}}{t_{i}+\left|y_{i}\right|}\right)^{n \lambda_{i}} \frac{d y_{1} d y_{2}}{t_{1}^{n} t_{2}^{m}} \frac{d x_{1} d t_{1}}{t_{1}} \\
\mathscr{R}_{2}\left(x_{2}, t_{2}\right)=\iint_{\widehat{I}} \iint_{\mathbb{R}^{n+m}}\left|\theta_{t_{1}, t_{2}}\left(\mathbf{1}_{(3 I)^{c}} \otimes h_{J_{1}}\right)(x-y)\right|^{2} \\
\cdot \prod_{i=1}^{2}\left(\frac{t_{i}}{t_{i}+\left|y_{i}\right|}\right)^{n \lambda_{i}} \frac{d y_{1} d y_{2}}{t_{1}^{n} t_{2}^{m}} \frac{d x_{1} d t_{1}}{t_{1}} \\
:=\iint_{\widehat{I}} H(x, t) \frac{d x_{1} d t_{1}}{t_{1}} .
\end{gathered}
$$

From combinations of Carleson and Hölder conditions and Lemma 5, it follows that 
Journal of Function Spaces

11

$$
\begin{aligned}
& \mathscr{R}_{1}\left(x_{2}, t_{2}\right) \lesssim\left|J_{1}\right|^{-1} \\
& \cdot \int_{\mathbb{R}^{m}} \iint_{\widehat{3 I}} \int_{\mathbb{R}^{n}}\left|\int_{J_{1}} \int_{3 I}\left[K_{t_{1}, t_{2}}\left(x-y,\left(z_{1}, z_{2}\right)\right)-K_{t_{1}, t_{2}}\left(x-y,\left(z_{1}, z_{2}+c_{J_{1}}\right)\right)\right] d z_{1} d z_{2}\right|^{2} \times\left(\frac{t_{1}}{t_{1}+\left|y_{1}\right|}\right)^{n \lambda_{1}} \frac{d y_{1}}{t_{1}^{n}} \frac{d x_{1} d t_{1}}{t_{1}} \\
& \cdot\left(\frac{t_{2}}{t_{2}+\left|y_{2}\right|}\right)^{m \lambda_{2}} \frac{d y_{2}}{t_{2}^{m} \leq\left|J_{1}\right|^{-1}} \\
& \cdot \int_{\mathbb{R}^{m}}\left[\int_{J_{1}}\left(\iint_{\widehat{3 I}} \int_{\mathbb{R}^{n}}\left|\int_{3 I}\left[K_{t_{1}, t_{2}}\left(x-y,\left(z_{1}, z_{2}\right)\right)-K_{t_{1}, t_{2}}\left(x-y,\left(z_{1}, z_{2}+c_{J_{1}}\right)\right)\right] d z_{1}\right|^{2} \times\left(\frac{t_{1}}{t_{1}+\left|y_{1}\right|}\right)^{n \lambda_{1}} \frac{d y_{1}}{t_{1}^{n}} \frac{d x_{1} d t_{1}}{t_{1}}\right)^{1 / 2} d z_{2}\right]^{2} \\
& \cdot\left(\frac{t_{2}}{t_{2}+\left|y_{2}\right|}\right)^{m \lambda_{2}} \frac{d y_{2}}{t_{2}^{m}} \leqslant|I|\left|J_{1}\right|^{-1} \int_{\mathbb{R}^{m}}\left(\int_{J_{1}} \frac{\ell\left(J_{1}\right)^{\beta} d z_{2}}{\left(t_{2}+\left|x_{2}-y_{2}-z_{2}\right|\right)^{m+\beta}}\right)^{2}\left(\frac{t_{2}}{t_{2}+\left|y_{2}\right|}\right)^{m \lambda_{2}} \frac{d y_{2}}{t_{2}^{m}} \lesssim|I| \\
& \cdot\left(\frac{\ell\left(J_{1}\right)^{\beta}\left|J_{1}\right|^{1 / 2}}{\left(\ell\left(J_{2}\right)+d\left(J_{1}, J_{2}\right)\right)^{m+\beta}}\right)^{2} \cdot
\end{aligned}
$$

The mixed Hölder and size estimate gives that

$$
\begin{aligned}
& \left|\theta_{t_{1}, t_{2}}\left(\mathbf{1}_{(3 I)^{c}} \otimes h_{J_{1}}\right)(x-y)\right| \\
& \leq\left|J_{1}\right|^{-1 / 2} \int_{(3 I)^{c}} \frac{t_{1}^{\alpha}}{\left(t_{1}+\left|x_{1}-y_{1}-z_{1}\right|\right)^{n+\alpha}} d z_{1} \\
& \quad \times \int_{J_{1}} \frac{\ell\left(J_{1}\right)^{\beta}}{\left(t_{2}+\left|x_{2}-y_{2}-z_{2}\right|\right)^{m+\beta}} d z_{2} .
\end{aligned}
$$

Thus, collecting the estimates of Lemma 5 and (55), one can deduce that

$$
\begin{aligned}
& H(x, t) \lesssim\left|J_{1}\right|^{-1} \int_{\mathbb{R}^{n}}\left(\int_{(3 I)^{c}} \frac{t_{1}^{\alpha} d z_{1}}{\left(t_{1}+\left|x_{1}-y_{1}-z_{1}\right|\right)^{n+\alpha}}\right)^{2} \\
& \cdot\left(\frac{t_{1}}{t_{1}+\left|y_{1}\right|}\right)^{n \lambda_{1}} \frac{d y_{1}}{t_{1}^{n}} \\
& \quad \times \int_{\mathbb{R}^{m}}\left(\int_{J_{1}} \frac{\ell\left(J_{1}\right)^{\beta} d z_{2}}{\left(t_{2}+\left|x_{2}-y_{2}-z_{2}\right|\right)^{m+\beta}}\right)^{2} \\
& \quad \cdot\left(\frac{t_{2}}{t_{2}+\left|y_{2}\right|}\right)^{m \lambda_{2}} \frac{d y_{2}}{t_{2}^{n}} \leqslant t_{1}^{2 \alpha} \ell(I)^{-2 \alpha}
\end{aligned}
$$

$$
\cdot\left(\frac{\ell\left(J_{1}\right)^{\beta}\left|J_{1}\right|^{1 / 2}}{\left(\ell\left(J_{2}\right)+d\left(J_{1}, J_{2}\right)\right)^{m+\beta}}\right)^{2} .
$$

Therefore, we obtain

$$
\begin{aligned}
& \mathscr{R}_{2}\left(x_{2}, t_{2}\right)=\iint_{\widehat{I}} H(x, t) \frac{d x_{1} d t_{1}}{t_{1}} \leqslant|I| \ell(I)^{-2 \alpha} \\
& \quad \cdot \int_{0}^{\ell(I)} t_{1}^{2 \alpha-1} d t_{1} \cdot\left(\frac{\ell\left(J_{1}\right)^{\beta}\left|J_{1}\right|^{1 / 2}}{\left(\ell\left(J_{2}\right)+d\left(J_{1}, J_{2}\right)\right)^{m+\beta}}\right)^{2} \lesssim|I| \\
& \cdot\left(\frac{\ell\left(J_{1}\right)^{\beta}\left|J_{1}\right|^{1 / 2}}{\left(\ell\left(J_{2}\right)+d\left(J_{1}, J_{2}\right)\right)^{m+\beta}}\right)^{2} .
\end{aligned}
$$

Thus, we finish the proof of Lemma 7.

Now we bound $\mathscr{G}_{\mathrm{Car},<}$. If $\ell\left(J_{1}\right)<\ell\left(J_{2}\right)$, then we have

$$
\mathscr{R}\left(x_{2}, t_{2}\right) \lesssim|I|\left(A_{J_{1}, J_{2}}\left|J_{2}\right|^{-1 / 2}\right)^{2} .
$$

Therefore, we obtain the following estimate

$$
\begin{aligned}
& \mathscr{G}_{\mathrm{Car},<}=\sum_{J_{2}: \text { good }} \iint_{W_{J_{2}}} \sum_{I: \text { good }} \iint_{W_{I}} \iint_{\mathbb{R}^{n+m}}\left|\sum_{J_{1}: \ell\left(J_{1}\right)<\ell\left(J_{2}\right)}\left\langle f_{J_{1}}\right\rangle_{I} \theta_{t_{1}, t_{2}}\left(\mathbf{1} \otimes h_{J_{1}}\right)(x-y)\right|^{2} \times\left(\frac{t_{1}}{t_{1}+\left|y_{1}\right|}\right)^{n \lambda_{1}}\left(\frac{t_{2}}{t_{2}+\left|y_{2}\right|}\right)^{m \lambda_{2}} \frac{d y_{1}}{t_{1}^{n}} \frac{d y_{2}}{t_{2}^{m}} \frac{d x_{1} d t_{1}}{t_{1}} \frac{d x_{2} d t_{2}}{t_{2}} \\
& \leq \sum_{J_{2}} \iint_{W_{J_{2}}} \sum_{I}\left[\sum_{J_{1}: \ell\left(J_{1}\right)<\ell\left(J_{2}\right)}\left(\iint_{W_{I}} \iint_{\mathbb{R}^{n+m}}\left|\left\langle f_{J_{1}}\right\rangle_{I} \theta_{t_{1}, t_{2}}\left(\mathbf{1} \otimes h_{J_{1}}\right)(x-y)\right|^{2} \times\left(\frac{t_{1}}{t_{1}+\left|y_{1}\right|}\right)^{n \lambda_{1}}\left(\frac{t_{2}}{t_{2}+\left|y_{2}\right|}\right)^{m \lambda_{2}} \frac{d y_{1}}{t_{1}^{n}} \frac{d y_{2}}{t_{2}^{m}} \frac{d x_{1} d t_{1}}{t_{1}}\right)^{1 / 2}\right]^{2} \frac{d x_{2} d t_{2}}{t_{2}}
\end{aligned}
$$




$$
\begin{aligned}
& \leq \sum_{J_{2}} \iint_{W_{I_{2}}}\left[\sum_{I_{1}: \ell\left(J_{1}\right)<\ell\left(J_{2}\right)}\left(\sum_{I}\left|\left\langle f_{I_{1}}\right\rangle_{I}\right|^{2} \iint_{W_{I}} \iint_{\mathbb{R}^{n+m}}\left|\theta_{t_{1}, t_{2}}\left(\mathbf{1} \otimes h_{J_{1}}\right)(x-y)\right|^{2} \times\left(\frac{t_{1}}{t_{1}+\left|y_{1}\right|}\right)^{n \lambda_{1}}\left(\frac{t_{2}}{t_{2}+\left|y_{2}\right|}\right)^{m \lambda_{2}} \frac{d y_{1}}{t_{1}^{n}} \frac{d y_{2}}{t_{2}^{m}} \frac{d x_{1} d t_{1}}{t_{1}}\right)^{1 / 2}\right]^{2} \frac{d x_{2} d t_{2}}{t_{2}} \\
& \leq \sum_{J_{2}}\left[\sum_{J_{1}: \ell\left(J_{1}\right)<\ell\left(J_{2}\right)} A_{J_{1} J_{2}}\left\|f_{J_{1}}\right\|_{L^{2}\left(\mathbb{R}^{n}\right)}\right]^{2} \leq \sum_{J_{1}}\left\|f_{J_{1}}\right\|_{L^{2}\left(\mathbb{R}^{n}\right)}^{2} \leq\|f\|_{L^{2}\left(\mathbb{R}^{n+m}\right)}^{2} .
\end{aligned}
$$

So far, we have completed the estimate of $\mathscr{G}_{\geq,<}$.

As for the term $\mathscr{G}_{<, \geq}$, it is completely symmetric with the term $\mathscr{G}_{\geq,<}$. It is worth noting that the mixed Hölder and size estimate and the combination of Carleson and Hölder estimate are symmetric, respectively. Thus the estimate for $\mathscr{G}_{<, \geq}$is also true and we here omit its proof.

\section{The Case: $\ell\left(I_{1}\right) \geq \ell\left(I_{2}\right)$ and $\ell\left(J_{1}\right) \geq \ell\left(J_{2}\right)$}

Similar to what we have done before, the summation $\ell\left(I_{1}\right) \geq$ $\ell\left(I_{2}\right)$ was decomposed into the separated, Nested, and adjacent terms. A similar splitting in the summation $\ell\left(J_{1}\right) \geq \ell\left(J_{2}\right)$ is also performed. This splits the whole summation into nine parts as follows:

$$
\begin{aligned}
\mathscr{G}_{\geq, \geq} \leq & \mathscr{G}_{\text {sep,sep }}+\mathscr{G}_{\text {sep,nes }}+\mathscr{G}_{\text {sep,adj }}+\mathscr{G}_{\text {nes,sep }} \\
& +\mathscr{G}_{\text {nes,nes }}+\mathscr{G}_{\text {nes,adj }}+\mathscr{G}_{\text {adj,sep }}+\mathscr{G}_{\text {adj,nes }} \\
& +\mathscr{G}_{\text {adj,adj }} .
\end{aligned}
$$

5.1. Nested/Nested: $\mathscr{G}_{\text {nes,nes }}$. We begin with the term $\mathscr{G}_{\text {nes,nes }}$, where the new biparameter phenomena will appear. Noting that although this is only one of the many cases one needs to discuss in order to obtain a full estimate for $\mathscr{G}_{\geq, \geq}$term all the main difficulties in other cases are in fact already embedded in Nested/Nested. The fact will become more and more clear throughout the proof. Similarly, for the singular integral operators including biparameter and multiparameter cases, the Nested part is also the most difficult one. Because it involves in some paraproduct estimates and all the BMO type estimates.

The decomposition of $h_{I^{(k)}}$ in (50) gives that

$$
\mathscr{G}_{\text {nes,nes }} \leqslant \mathscr{G}_{\text {mod,mod }}+\mathscr{G}_{\mathrm{Car}, \mathrm{Car}}+\mathscr{G}_{\text {mod,Car }}+\mathscr{G}_{\mathrm{Car} \text { mod }} \text {, }
$$

where

$$
\begin{aligned}
& \mathscr{G}_{\text {mod,mod }} \\
& \left.=\sum_{I, J \text { : good }} \iint_{W_{J}} \iint_{W_{I}} \iint_{\mathbb{R}^{n+m}} \mid \sum_{k=1}^{\infty} \sum_{i=1}^{\infty} f_{I^{(k)}}\right)\left.^{(i)} \theta_{t_{1}, t_{2}}\left(s_{I}^{k} \otimes s_{J}^{i}\right)(x-y)\right|^{2} \times\left(\frac{t_{1}}{t_{1}+\left|y_{1}\right|}\right)^{n \lambda_{1}}\left(\frac{t_{2}}{t_{2}+\left|y_{2}\right|}\right)^{m \lambda_{2}} \frac{d y_{1}}{t_{1}^{n}} \frac{d y_{2}}{t_{2}^{m}} \frac{d x_{1} d t_{1}}{t_{1}} \frac{d x_{2} d t_{2}}{t_{2}}, \\
& \mathscr{G}_{\text {mod,Car }} \\
& =\sum_{I, J \text { :good }} \iint_{W_{J}} \iint_{W_{I}} \iint_{\mathbb{R}^{n+m}}\left|\sum_{k=1}^{\infty}\left\langle f_{I^{(k)}}\right\rangle_{J} \theta_{t_{1}, t_{2}}\left(s_{I}^{k} \otimes \mathbf{1}\right)(x-y)\right|^{2} \times\left(\frac{t_{1}}{t_{1}+\left|y_{1}\right|}\right)^{n \lambda_{1}}\left(\frac{t_{2}}{t_{2}+\left|y_{2}\right|}\right)^{m \lambda_{2}} \frac{d y_{1}}{t_{1}^{n}} \frac{d y_{2}}{t_{2}^{m}} \frac{d x_{1} d t_{1}}{t_{1}} \frac{d x_{2} d t_{2}}{t_{2}}, \\
& \mathscr{G}_{\text {Car,mod }} \sum_{I, J \text { :good }} \iint_{W_{J}} \iint_{W_{I}} \iint_{\mathbb{R}^{n+m}}\left|\sum_{\ell=1}^{\infty}\left\langle f_{J^{(i)}}\right\rangle_{I} \theta_{t_{1}, t_{2}}\left(\mathbf{1} \otimes s_{J}^{i}\right)(x-y)\right|^{2} \times\left(\frac{t_{1}}{t_{1}+\left|y_{1}\right|}\right)^{n \lambda_{1}}\left(\frac{t_{2}}{t_{2}+\left|y_{2}\right|}\right)^{m \lambda_{2}} \frac{d y_{1}}{t_{1}^{n}} \frac{d y_{2}}{t_{2}^{m}} \frac{d x_{1} d t_{1}}{t_{1}} \frac{d x_{2} d t_{2}}{t_{2}}, \\
& \mathscr{G}_{\text {Car,Car }}=\sum_{I, J: \text { good }}\left|\langle f\rangle_{I \times J}\right|^{2} \iint_{W_{J}} \iint_{W_{I}} \iint_{\mathbb{R}^{n+m}}\left|\theta_{t_{1}, t_{2}}(\mathbf{1})(x-y)\right|^{2} \times\left(\frac{t_{1}}{t_{1}+\left|y_{1}\right|}\right)^{n \lambda_{1}}\left(\frac{t_{2}}{t_{2}+\left|y_{2}\right|}\right)^{m \lambda_{2}} \frac{d y_{1}}{t_{1}^{n}} \frac{d y_{2}}{t_{2}^{m}} \frac{d x_{1} d t_{1}}{t_{1}} \frac{d x_{2} d t_{2}}{t_{2}} .
\end{aligned}
$$

5.1.1. Estimate of $\mathscr{G}_{\text {mod,mod }}$. We proceed using the standard argument as in Lemma 6 . The size condition and (55) lead to the bound

$$
\left(\iint_{\mathbb{R}^{n+m}}\left|\theta_{t_{1}, t_{2}}\left(s_{I}^{k} \otimes s_{J}^{i}\right)(x-y)\right|^{2}\left(\frac{t_{1}}{t_{1}+\left|y_{1}\right|}\right)^{n \lambda_{1}}\right.
$$

$$
\begin{aligned}
& \left.\cdot\left(\frac{t_{2}}{t_{2}+\left|y_{2}\right|}\right)^{m \lambda_{2}} \frac{d y_{1}}{t_{1}^{n}} \frac{d y_{2}}{t_{2}^{m}}\right)^{1 / 2} \\
& \leq 2^{-\alpha k / 2}\left|I^{(k)}\right|^{-1 / 2} \cdot 2^{-\beta i}\left|J^{(i)}\right|^{-1 / 2} .
\end{aligned}
$$


It is similar to estimate $\mathscr{G}_{\text {mod, }<}$ to analyze $\mathscr{G}_{\text {mod,mod }}$.

$$
\begin{aligned}
& \mathscr{G}_{\text {mod,mod }} \\
& \leq \sum_{k, i} 2^{-\alpha k / 2} 2^{-\beta i} \sum_{Q, R}\left|f_{Q R}\right|^{2} \frac{1}{|Q|} \sum_{I: I^{(k)}=Q}|I| \cdot \frac{1}{|R|} \sum_{J: J^{(i)}=R}|J| \\
& \quad \leqslant\|f\|_{L^{2}\left(\mathbb{R}^{n+m}\right)}^{2} .
\end{aligned}
$$

5.1.2. Estimate of $\mathscr{G}_{\text {Car,Car }}$. Applying the biparameter Carleson condition, it immediately yields that

$$
\begin{aligned}
\mathscr{G}_{\mathrm{Car}, \mathrm{Car}} & =\sum_{I, J}\left|\langle f\rangle_{I \times J}\right|^{2} C_{I J}^{\mathscr{D}}=2 \int_{0}^{\infty} \sum_{\substack{I, J \\
\left|\langle f\rangle_{I \times J}\right|>t}} C_{I J}^{\mathscr{D}} t d t \\
& \lesssim \int_{0}^{\infty} \sum_{\substack{I, J \\
I \times J \subset\left\{M_{\mathscr{D}} f>t\right\}}} C_{I J}^{\mathscr{D}} t d t \\
& \lesssim \int_{0}^{\infty}\left|\left\{M_{\mathscr{D}} f>t\right\}\right| t d t \lesssim\left\|M_{\mathscr{D}} f\right\|_{L^{2}\left(\mathbb{R}^{n+m}\right)}^{2} \\
& \lesssim\|f\|_{L^{2}\left(\mathbb{R}^{n+m}\right)}^{2},
\end{aligned}
$$

$$
\begin{aligned}
& \mathscr{G}_{\text {Car,mod }} \\
& \leq \sum_{J: \text { good }} \iint_{W_{J}} \sum_{I}\left[\sum_{i=1}^{\infty}\left|\left\langle f_{J^{(i)}}\right\rangle_{I}\right|\left(\iint_{W_{I}} \iint_{\mathbb{R}^{n+m}}\left|\theta_{t_{1}, t_{2}}\left(1 \otimes s_{J}^{i}\right)(x-y)\right|^{2} \times\left(\frac{t_{1}}{t_{1}+\left|y_{1}\right|}\right)^{n \lambda_{1}}\left(\frac{t_{2}}{t_{2}+\left|y_{2}\right|}\right)^{m \lambda_{2}} \frac{d y_{1}}{t_{1}^{n}} \frac{d y_{2}}{t_{2}^{m}} \frac{d x_{1} d t_{1}}{t_{1}}\right)^{1 / 2}\right]^{2} \frac{d x_{2} d t_{2}}{t_{2}} \\
& \lesssim \sum_{i=1}^{\infty} 2^{-\beta i / 2} \sum_{R}\left\|f_{R}\right\|_{L^{2}\left(\mathbb{R}^{n}\right)}^{2} \frac{1}{|R|} \sum_{J: J^{(i)=R}}|I| \lesssim\|f\|_{L^{2}\left(\mathbb{R}^{n+m}\right)}^{2} .
\end{aligned}
$$

5.2. The Rest of Terms. As for the estimates of the remaining terms, they are simply combinations of the techniques we have used above. Thereby, we here only present certain key points.

When reviewing the above proof, one will realize that the central part is to dominate $\mathscr{P}(x, t), \mathcal{Q}(x, t)$, and $\mathscr{R}\left(x_{2}, t_{2}\right)$. So do the rest of terms. Moreover, the initial estimates of $\mathscr{P}$, $Q$, and $\mathscr{R}$ are retained in the inequality (33) and Lemmas 6 and 7 , respectively. They do not involve the relationship of side length of cubes $I_{1}, I_{2}, J_{1}$, and $J_{2}$. Thus, based on the inequality (33) and Lemmas 6 and 7, one only needs to add the corresponding the relationship of side length.

Consequently, using the size condition or the mixed Hölder and size condition, it yields the bounds for $\mathscr{G}_{\text {sep,sep}}$, $\mathscr{G}_{\text {sep,adj }}, \mathscr{G}_{\text {adj,adj, }}$ and $\mathscr{G}_{\text {adj,sep }}$ directly. Finally, for the terms $\mathscr{G}_{\text {nes,sep }}$ and $\mathscr{G}_{\text {nes,adj }}$, nes is split into mod and Car. Applying the size condition and the combinations of Carleson and size estimate, we will bound them. The terms $\mathscr{G}_{\text {sep,nes }}$ and $\mathscr{G}_{\text {adj,nes }}$ are symmetric with respect to them, respectively. where in the last step we have used the $L^{p}(1<p<\infty)$ boundedness of the strong maximal function associated with rectangles.

\subsubsection{Estimate of $\mathscr{G}_{\mathrm{Car} \text { mod }}$ and $\mathscr{G}_{\mathrm{mod}, \mathrm{Car}}$}

Lemma 8. Let $J \in \mathscr{D}_{\text {m,good }},\left(x_{2}, t_{2}\right) \in W_{J_{2}}$ and $i \in \mathbb{N}$ be fixed. Then the Carleson condition is satisfied as follows:

$$
\begin{gathered}
\sum_{I^{\prime} \subset I} \iint_{W_{I^{\prime}}} \iint_{\mathbb{R}^{n+m}}\left|\theta_{t_{1}, t_{2}}\left(\mathbf{1} \otimes s_{J}^{i}\right)(x-y)\right|^{2} \\
\cdot\left(\frac{t_{1}}{t_{1}+\left|y_{1}\right|}\right)^{n \lambda_{1}} \\
\cdot\left(\frac{t_{2}}{t_{2}+\left|y_{2}\right|}\right)^{m \lambda_{2}} \frac{d y_{1}}{t_{1}^{n}} \frac{d y_{2}}{t_{2}^{m}} \frac{d x_{1} d t_{1}}{t_{1}} \\
\quad \leq 2^{-\beta i}|I| \cdot\left|J^{(i)}\right|^{-1} .
\end{gathered}
$$

Proof. The proof of Lemma 8 is similar to Lemma 7. The size condition and mixed Carleson and size estimate are used. In addition, the inequality (55) is used twice.

Therefore, $\mathscr{G}_{\text {Car,mod }}$ is bounded as below.

\section{The Necessity of Biparameter Carleson Condition}

We here show that the biparameter Carleson condition is necessary for $g_{\lambda_{1}, \lambda_{2}}^{*}$-function bound on $L^{2}\left(\mathbb{R}^{n+m}\right)$.

Suppose that $\theta_{t_{1}, t_{2}}=\theta_{t_{1}}^{n} \otimes \theta_{t_{2}}^{m}$ is bounded on $L^{2}\left(\mathbb{R}^{n+m}\right)$, where $\theta_{t_{1}}^{n}$ has a kernel $s_{t_{1}}^{n}\left(x_{1}, y_{1}\right)$ and $\theta_{t_{2}}^{n}$ has a kernel $s_{t_{2}}^{m}\left(x_{2}, y_{2}\right), x_{1}, y_{1} \in \mathbb{R}^{n}, x_{2}, y_{2} \in \mathbb{R}^{m}$, and $t_{1}, t_{2}>0$. We assume that these satisfy the size condition and the corresponding $L^{2}$ bounds in $\mathbb{R}^{n}$ and $\mathbb{R}^{m}$. We shall show that the biparameter Carleson condition (12) holds.

Define $\widetilde{\Omega}=\left\{M_{\mathscr{D}} \mathbf{1}_{\Omega}>1 / 2\right\}$ and $\widehat{\Omega}=\left\{M \mathbf{1}_{\widetilde{\Omega}}>c\right\}$ for a small enough dimensional constant $c=c(n, m)$, where $M_{\mathscr{D}}$ denotes the strong maximal function related to the grid $\mathscr{D}$ and $M$ denotes the strong maximal function. From the endpoint estimates for $M$ and $M_{\mathscr{D}}$, it follows that $|\widehat{\Omega}| \lesssim|\widetilde{\Omega}| \lesssim$ $|\Omega|$. Hence, it is enough to show that 


$$
\sum_{\substack{I \times J \in \mathscr{D} \\ I \times J \subset \Omega}} \iint_{W_{J}} \iint_{W_{I}} \iint_{\mathbb{R}^{n+m}}\left|\theta_{t_{1}, t_{2}} \mathbf{1}_{\widehat{\Omega}^{c}}\left(y_{1}, y_{2}\right)\right|^{2}\left(\frac{t_{1}}{t_{1}+\left|x_{1}-y_{1}\right|}\right)^{n \lambda_{1}} \times\left(\frac{t_{2}}{t_{2}+\left|x_{2}-y_{2}\right|}\right)^{m \lambda_{2}} \frac{d y_{1}}{t_{1}^{n}} \frac{d y_{2}}{t_{2}^{m}} \frac{d x_{1} d t_{1}}{t_{1}} \frac{d x_{2} d t_{2}}{t_{2}} \leq|\Omega| .
$$

For every $J \in \mathscr{D}_{m}$ we let $\mathscr{F}_{J}$ consist of the maximal $F \in \mathscr{D}_{n}$ for which $F \times J \subset \widetilde{\Omega}$. Then we define $F_{J}:=\bigcup_{F \in \mathscr{F}_{I}} 2 F$. Moreover, for fixed $I \in \mathscr{D}_{n}$, let $\mathscr{G}_{I}$ be the family of the maximal $G \in \mathscr{D}_{m}$, for which $I \times G \subset \Omega$, and $I_{G} \in \mathscr{D}_{n}$ be the maximal cube for which $I_{G} \supset I$ and $I_{G} \times G \subset \widetilde{\Omega}$. So, we only need to show the following inequalities:

$$
\begin{aligned}
& \mathscr{G}_{1} \\
& =\sum_{\substack{I \times J \in \mathscr{D} \\
I \times J \subset \Omega}} \iint_{W_{J}} \iint_{W_{I}} \iint_{\mathbb{R}^{n+m}}\left|\theta_{t_{1}, t_{2}}\left(\mathbf{1}_{\widehat{\Omega}^{c}} \mathbf{1}_{F_{J}}\right)\left(y_{1}, y_{2}\right)\right|^{2}\left(\frac{t_{1}}{t_{1}+\left|x_{1}-y_{1}\right|}\right)^{n \lambda_{1}} \times\left(\frac{t_{2}}{t_{2}+\left|x_{2}-y_{2}\right|}\right)^{m \lambda_{2}} \frac{d y_{1}}{t_{1}^{n}} \frac{d y_{2}}{t_{2}^{m}} \frac{d x_{1} d t_{1}}{t_{1}} \frac{d x_{2} d t_{2}}{t_{2}} \\
& :=\sum_{J} \iint_{W_{J}} \mathscr{G}_{J}\left(x_{2}, t_{2}\right) \frac{d x_{2} d t_{2}}{t_{2}} \lesssim|\Omega|, \\
& \mathscr{G}_{2} \\
& =\sum_{\substack{I \times J \in \mathscr{D} \\
I \times J \subset \Omega}} \iint_{W_{J}} \iint_{W_{I}} \iint_{\mathbb{R}^{n+m}}\left|\theta_{t_{1}, t_{2}}\left(\mathbf{1}_{\widehat{\Omega}^{c}} \mathbf{1}_{F_{J}^{c}}\right)\left(y_{1}, y_{2}\right)\right|^{2}\left(\frac{t_{1}}{t_{1}+\left|x_{1}-y_{1}\right|}\right)^{n \lambda_{1}} \times\left(\frac{t_{2}}{t_{2}+\left|x_{2}-y_{2}\right|}\right)^{m \lambda_{2}} \frac{d y_{1}}{t_{1}^{n}} \frac{d y_{2}}{\frac{t_{2}^{m}}{\frac{d x_{1}}{t_{1}}}} \frac{d t_{1}}{t_{1}} \frac{d x_{2} d t_{2}}{t_{2}} \\
& :=\sum_{I} \iint_{W_{I}} \mathscr{G}_{I}\left(x_{1}, t_{1}\right) \frac{d x_{1} d t_{1}}{t_{1}} \lesssim|\Omega| .
\end{aligned}
$$

To attain the goal, we need to first estimate $\mathscr{G}_{J}\left(x_{2}, t_{2}\right)$ and $\mathscr{G}_{I}\left(x_{1}, t_{1}\right)$. Actually, Minkowski's integral inequality and size estimate yield that

$$
\begin{aligned}
& \mathscr{G}_{J}\left(x_{2}, t_{2}\right) \\
& \leq\left[\int_{\mathbb{R}^{m}}\left(\iint_{\mathbb{R}_{+}^{n+1}} \iint_{\mathbb{R}^{n+m}}\left|K_{t_{2}}^{m}\left(y_{2}, z_{2}\right)\right|^{2}\left|\theta_{t_{1}, t_{2}}\left(\mathbf{1}_{\Omega^{c}} \mathbf{1}_{F_{J}}\right)\left(y_{1}\right)\right|^{2}\left(\frac{t_{1}}{t_{1}+\left|x_{1}-y_{1}\right|}\right)^{n \lambda_{1}} \times\left(\frac{t_{2}}{t_{2}+\left|x_{2}-y_{2}\right|}\right)^{m \lambda_{2}} \frac{d y_{1}}{t_{1}^{n}} \frac{d y_{2}}{t_{2}^{m}} \frac{d x_{1} d t_{1}}{t_{1}}\right)^{1 / 2} d z_{2}\right]^{2} \\
& \leq\left[\int_{\mathbb{R}^{m}}\left(\int_{\mathbb{R}^{m}}\left(\frac{t_{2}^{\beta}}{\left(t_{2}+\left|y_{2}-z_{2}\right|\right)^{m+\beta}}\right)^{2}\left(\frac{t_{2}}{t_{2}+\left|x_{2}-y_{2}\right|}\right)^{m \lambda_{2}} \frac{d y_{2}}{t_{2}^{m}}\right)^{1 / 2} \times\left\|\mathbf{1}_{\widehat{\Omega}^{c}}\left(\cdot, z_{2}\right) \mathbf{1}_{F_{J}}\right\|_{L^{2}\left(\mathbb{R}^{n}\right)} d z_{2}\right]^{2} \\
& \leq\left[\int_{\mathbb{R}^{m}} \frac{\ell(J)^{\beta}}{\left(\ell(J)+\left|x_{2}-z_{2}\right|\right)^{m+\beta}}\left\|\mathbf{1}_{\widehat{\Omega}^{c}}\left(\cdot, z_{2}\right) \mathbf{1}_{F_{J}}\right\|_{L^{2}\left(\mathbb{R}^{n}\right)} d z_{2}\right]^{2} \leq \int_{\mathbb{R}^{m}} \frac{\ell(J)^{\beta}}{\left(\ell(J)+\left|x_{2}-z_{2}\right|\right)^{m+\beta}\left\|\mathbf{1}_{\widehat{\Omega}^{c}}\left(\cdot, z_{2}\right) \mathbf{1}_{F_{J}}\right\|_{L^{2}\left(\mathbb{R}^{n}\right)}^{2} d z_{2}} \\
& \leq \int_{\mathbb{R}^{m}} \frac{\ell(J)^{\beta}}{\left|z_{2}-c_{J}\right|^{m+\beta}}\left\|\mathbf{1}_{\widehat{\Omega}^{c}}\left(\cdot, z_{2}\right) \mathbf{1}_{F_{J}}\right\|_{L^{2}\left(\mathbb{R}^{n}\right)}^{2} d z_{2}=\int_{\mathbb{R}^{n}} \mathbf{1}_{F_{J}}\left(z_{1}\right) \int_{\mathbb{R}^{m}} \frac{\ell(J)^{\beta}}{\left|z_{2}-c_{J}\right|^{m+\beta}} \mathbf{1}_{\widehat{\Omega}^{c}}\left(z_{1}, z_{2}\right) d z_{2} d z_{1} .
\end{aligned}
$$




$$
\begin{aligned}
& \mathscr{G}_{I}\left(x_{1}, t_{1}\right) \\
& \quad=\sum_{G \in \mathscr{G}_{I}} \sum_{J: J \subset G} \iint_{W_{J}} \iint_{\mathbb{R}^{n+m}}\left|\theta_{t_{1}, t_{2}}\left(\mathbf{1}_{\widehat{\Omega}^{c}} \mathbf{1}_{F_{J}^{c}}\right)\left(y_{1}, y_{2}\right)\right|^{2}\left(\frac{t_{1}}{t_{1}+\left|x_{1}-y_{1}\right|}\right)^{n \lambda_{1}} \times\left(\frac{t_{2}}{t_{2}+\left|x_{2}-y_{2}\right|}\right)^{m \lambda_{2}} \frac{d y_{1}}{t_{1}^{n}} \frac{d y_{2}}{t_{2}^{m}} \frac{d x_{2} d t_{2}}{t_{2}} \\
& \quad \leq\left[\int_{\mathbb{R}^{n}} \frac{\ell(I)^{\alpha}}{\left(\ell(I)+\left|x_{1}-z_{1}\right|\right)^{n+\alpha}}\left(\sum_{G \in \mathscr{G}_{I}} \mathbf{1}_{\left(2 I_{G}\right)^{c}}\left(z_{1}\right)|G|\right)^{1 / 2} d z_{1}\right]^{2} \lesssim \sum_{G \in \mathscr{G}_{I}}|G| \int_{\mathbb{R}^{n}} \frac{\ell(I)^{\alpha}}{\left(\ell(I)+\left|x_{1}-z_{1}\right|\right)^{n+\alpha}} \mathbf{1}_{\left(2 I_{G}\right)^{c}}\left(z_{1}\right) d z_{1} \\
& \quad \leq \sum_{G \in \mathscr{G}_{I}}|G| \int_{I_{G}^{c}} \frac{\ell(I)^{\alpha}}{\left|z_{1}-c_{I_{G}}\right|^{n+\alpha}} d z_{1} \lesssim \sum_{G \in \mathscr{G}_{I}}|G|\left(\frac{\ell(I)}{\ell\left(I_{G}\right)}\right)^{\alpha} .
\end{aligned}
$$

The remaining calculation is a routine application of the idea of [14]. We here omit the details. Finally, we obtain

$$
\begin{aligned}
& \mathscr{G}_{1}=\sum_{J} \iint_{W_{J}} \mathscr{G}_{J}\left(x_{2}, t_{2}\right) \frac{d x_{2} d t_{2}}{t_{2}} \leqslant|\Omega|, \\
& \mathscr{G}_{2}=\sum_{I} \iint_{W_{I}} \mathscr{G}_{I}\left(x_{1}, t_{1}\right) \frac{d x_{1} d t_{1}}{t_{1}} \leqslant|\Omega| .
\end{aligned}
$$

Thus, we have proved the necessity.

\section{Competing Interests}

The authors declare that they have no competing interests.

\section{Acknowledgments}

The second author was supported partly by NSFC (nos. 11471041 and 11671039), the Fundamental Research Funds for the Central Universities (no. 2014KJJCA10), and NCET-130065 .

\section{References}

[1] J. Littlewood and R. Paley, "Theorems on Fourier series and power series, II," Proceedings London Mathematical Society, vol. 42, pp. 52-89, 1936.

[2] E. M. Stein, Singular Integrals and Differentiability Properties of Functions, Princeton Mathematical Series, No. 30, Princeton University Press, Princeton, NJ, USA, 1970.

[3] E. M. Stein, "On some funcions of Littlewood-Paley and Zygmund," Bulletin of the American Mathematical Society, vol. 67, pp. 99-101, 1961.

[4] C. Fefferman, "Inequalities for strongly singular convolution operators," Acta Mathematica, vol. 124, pp. 9-36, 1970.

[5] M. Cao, K. Li, and Q. Xue, "A characterization of two weight norm inequality for Littlewood-Paley $\mathbf{g}_{\lambda}^{*}$-function," http://arxiv.org/abs/1504.07850.

[6] M. T. Lacey, "The two weight inequality for the Hilbert transform: a primer," https://arxiv.org/abs/1304.5004.

[7] M. T. Lacey, "Two-weight inequality for the Hilbert transform: a real variable characterization, II," Duke Mathematical Journal, vol. 163, no. 15, pp. 2821-2840, 2014.
[8] M. T. Lacey and K. Li, "Two weight norm inequalities for $g$ function," Mathematical Research Letters, vol. 21, no. 3, pp. 521536, 2014.

[9] M. T. Lacey, E. T. Sawyer, C.-Y. Shen, and I. Uriarte-Tuero, "Two-weight inequality for the Hilbert transform: a real variable characterization, I," Duke Mathematical Journal, vol. 163, no. 15, pp. 2795-2820, 2014.

[10] M. Cao and Q. Xue, "A non-homogeneous local Tb theorem for Littlewood-Paley $g_{\lambda}^{*}$-function with Lptesting condition," https://arxiv.org/abs/1507.05291.

[11] R. Fefferman and E. M. Stein, "Singular integrals on product spaces," Advances in Mathematics, vol. 45, no. 2, pp. 117-143, 1982.

[12] H. Martikainen, "Representation of bi-parameter singular integrals by dyadic operators," Advances in Mathematics, vol. 229, no. 3, pp. 1734-1761, 2012.

[13] T. Hytönen and H. Martikainen, "Non-homogeneous T1 theorem for bi-parameter singular integrals," Advances in Mathematics, vol. 261, pp. 220-273, 2014.

[14] H. Martikainen, "Boundedness of a class of bi-parameter square functions in the upper half-space," Journal of Functional Analysis, vol. 267, no. 10, pp. 3580-3597, 2014.

[15] Y. Ou, "A T(b) theorem on product spaces," Transactions of the American Mathematical Society, vol. 367, no. 9, pp. 6159-6197, 2015.

[16] T. P. Hytönen, "The sharp weighted bound for general Calderón-Zygmund operators," Annals of Mathematics, vol. 175, no. 3, pp. 1473-1506, 2012.

[17] F. Nazarov, S. Treil, and A. Volberg, "The Tb-theorem on nonhomogeneous spaces," Acta Mathematica, vol. 190, no. 2, pp. 151239, 2003. 


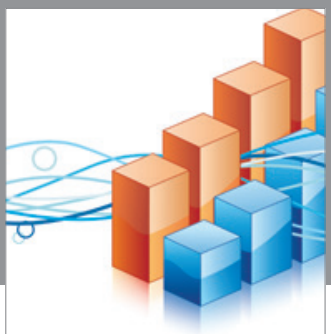

Advances in

Operations Research

vatem alat4

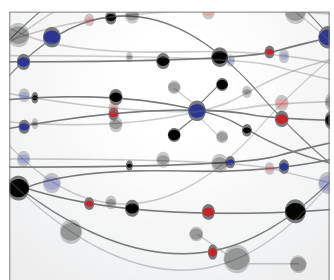

\section{The Scientific} World Journal
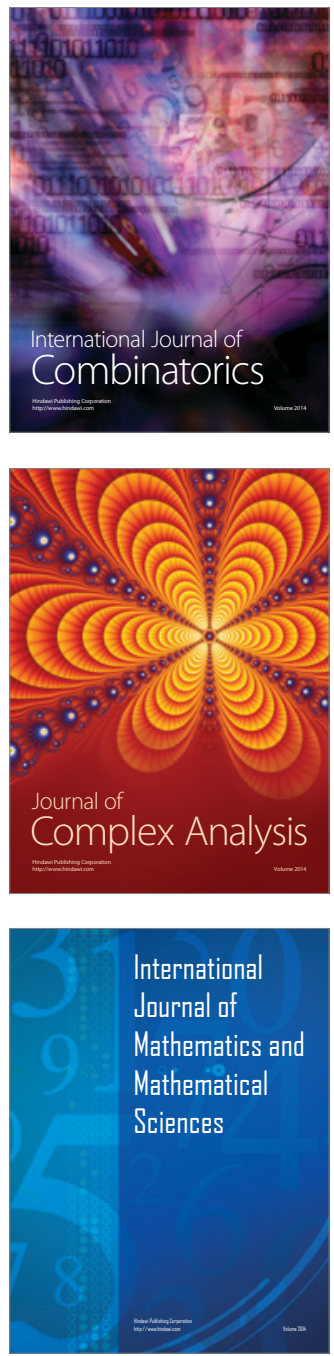
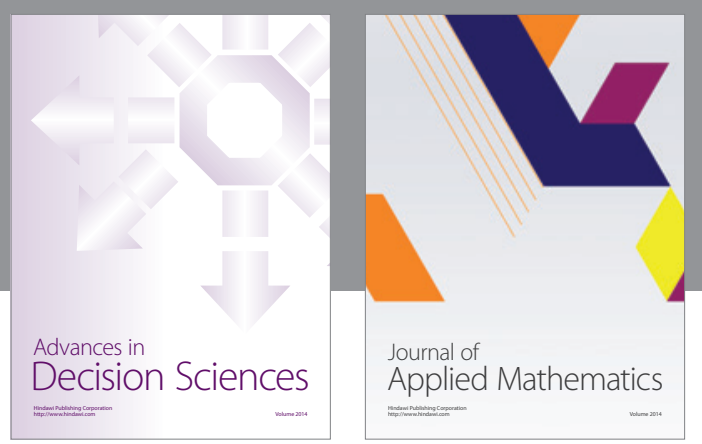

Algebra

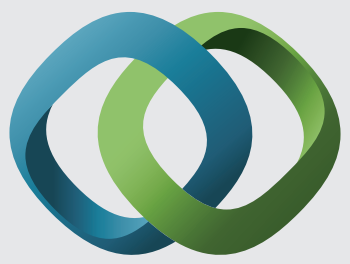

\section{Hindawi}

Submit your manuscripts at

http://www.hindawi.com
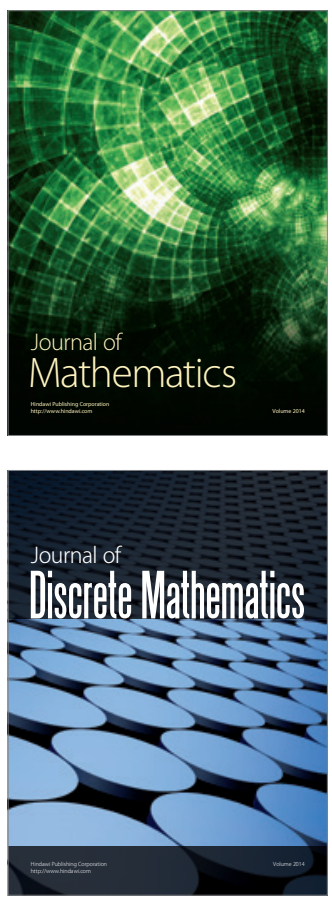

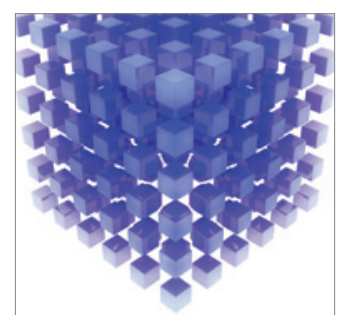

Mathematical Problems in Engineering
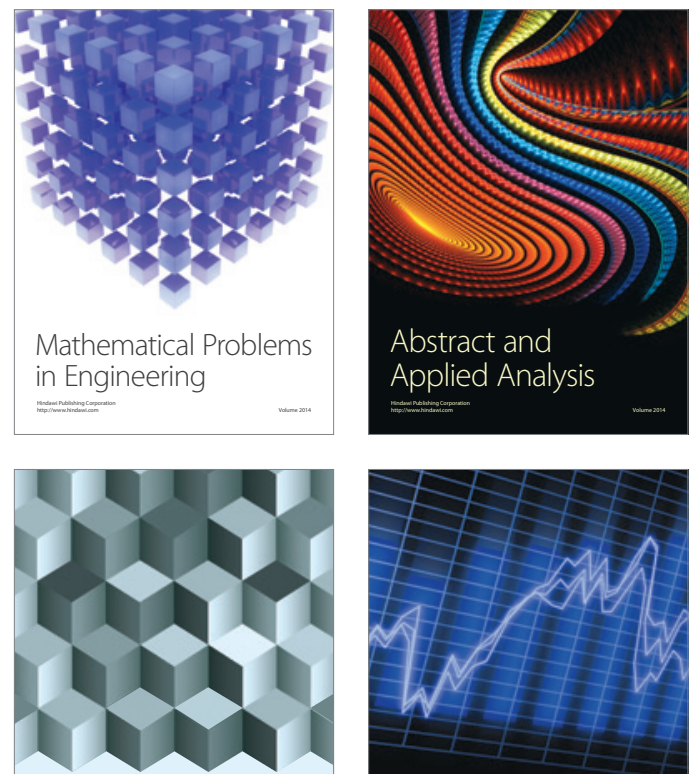

Journal of

Function Spaces

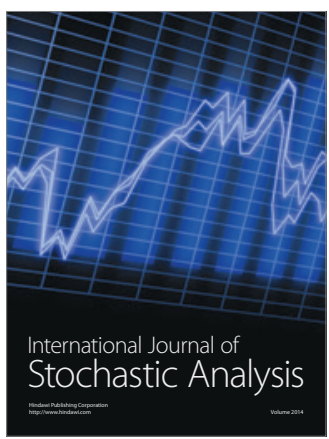

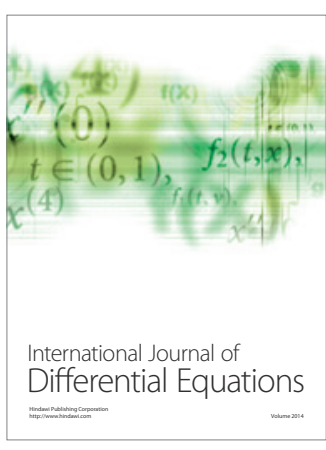
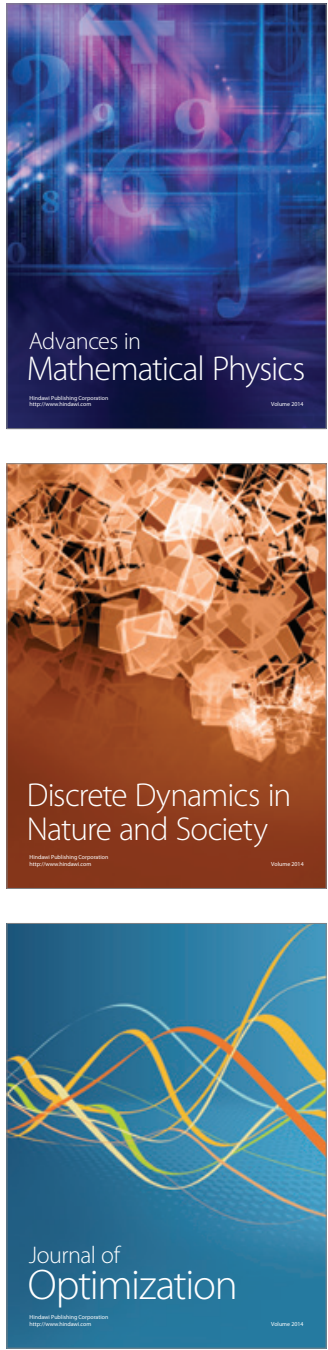